\title{
Manifolds with singularities accepting a metric of positive scalar curvature
}

\author{
BORIS BOTVINNIK \\ Department of Mathematics, University of Oregon \\ Eugene, OR 97403, USA \\ Email: botvinn@math.uoregon.edu
}

\begin{abstract}
We study the question of existence of a Riemannian metric of positive scalar curvature metric on manifolds with the Sullivan-Baas singularities. The manifolds we consider are Spin and simply connected. We prove an analogue of the Gromov-Lawson Conjecture for such manifolds in the case of particular type of singularities. We give an affirmative answer when such manifolds with singularities accept a metric of positive scalar curvature in terms of the index of the Dirac operator valued in the corresponding " $K$-theories with singularities". The key ideas are based on the construction due to Stolz, some stable homotopy theory, and the index theory for the Dirac operator applied to the manifolds with singularities. As a side-product we compute homotopy types of the corresponding classifying spectra.
\end{abstract}

AMS Classification numbers Primary: 57R15

Secondary: 53C21, 55T15, 57R90

Keywords: Positive scalar curvature, Spin manifolds, manifolds with singularities, Spin cobordism, characteristic classes in $K$-theory, cobordism with singularities, Dirac operator, $K$-theory with singularities, Adams spectral sequence, $\mathcal{A}(1)$-modules.

Proposed: Ralph Cohen

Seconded: Haynes Miller, Steven Ferry
Received: 2 November 1999

Revised: 28 August 2001

(C) Geometry $\&$ Topology Publications 


\section{Introduction}

1.1 Motivation It is well-known that the question of existence of positive scalar curvature metric is hard enough for regular manifolds. This question was studied extensively, and it is completely understood, see [9], [29], for simply connected manifolds and for manifolds with few particular fundamental groups, see [4], and also [23], [24] for a detailed discussion. At the same time, the central statement in this area, the Gromov-Lawson-Rosenberg Conjecture is known to be false for some particular manifolds, see [26]. To motivate our interest we first address a couple of naive questions. We shall consider manifolds with boundary, and we always assume that a metric on a manifold is product metric near its boundary. We use the abbreviation "psc" for "positive scalar curvature" throughout the paper.

Let $\left(P, g_{P}\right)$ be a closed Riemannian manifold, where the metric $g_{P}$ is not assumed to be of positive scalar curvature. Let $X$ be a closed manifold, such that the product $X \times P$ is a boundary of a manifold $Y$.

Naive Question 1 Does there exist a psc-metric $g_{X}$ on $X$, so that the product metric $g_{X} \times g_{P}$ could be extended to a psc-metric $g_{Y}$ on $Y$ ?

Examples (1) Let $P=$ $\langle k\rangle=\{k$ points $\}$, then a manifold $Y$ with $\partial Y=X \times\langle k\rangle$ is called a $\mathbf{Z} / k$-manifold. When $k=1$ (or $X=\partial Y$ ) the above question is essentially trivial. Say, if $X$ and $Y$ are simply connected Spin manifolds, and $\operatorname{dim} X=n-1 \geq 5$, there is always a psc-metric $g_{X}$ which could be extended to a

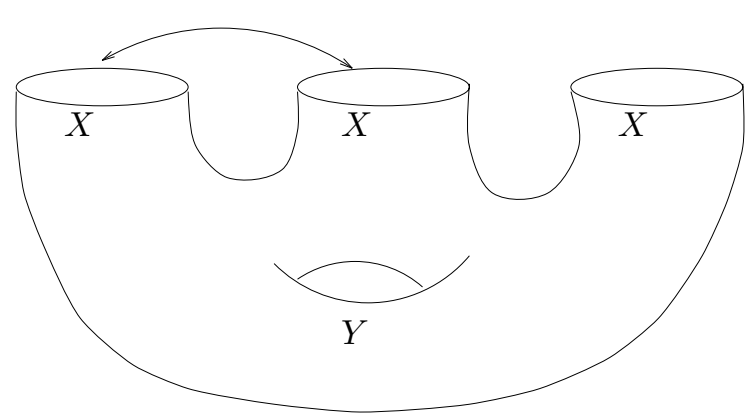

Figure 1: $\mathbf{Z} / k-$ manifold psc-metric $g_{Y}$.

To see this one can delete a small open disk $D^{n} \subset Y$, and then push the standard metric on $S^{n-1}$ through the cobordism $W=Y \backslash D^{n}$ to the manifold $X$ using the surgery technique due to Gromov, Lawson [9] and Schoen, Yau $[27]$.

(2) The case $P=\langle k\rangle$ with $k \geq 2$ is not as simple. For example, there are many simply connected Spin manifolds $X$ of dimension $4 k$ (for most $k$ ) which are not cobordant to zero, and, in the same time, two copies of $X$ are. Let 
$\partial Y=2 X$. It is not obvious that one can find a psc-metric $g_{X}$ on $X$, so that the product metric $g_{X} \times\langle 2\rangle$ extends to a psc-metric $g_{Y}$ on $Y$.

(3) Let $\Sigma^{m}$ (where $m=8 l+1$ or $8 l+2$, and $l \geq 1$ ) be a homotopy sphere which does not admit a psc-metric, see [12]. We choose $k \geq 2$ disjoint discs $D_{1}^{m}, \ldots, D_{k}^{m} \subset \Sigma^{m}$ and delete their interior. The resulting manifold $Y^{m}$ has the boundary $S^{m-1} \times\langle k\rangle$. Clearly it is not possible to extend the standard metrics on the spheres $S^{m-1} \times\langle k\rangle$ to a psc-metric on the manifold $Y$ since otherwise it would give a psc-metric on the original homotopy sphere $\Sigma^{m}$. However, it is not obvious that for any choice of a psc-metric $g$ on $S^{m-1}$ the metric $g \times\langle k\rangle$ could not be extended to a psc-metric on $Y^{m}$.

(4) Let $P$ be again $k$ points. Consider a Joyce manifold $J^{8}$ (Spin, simply connected, Ricci flat, with $\hat{A}\left(J^{8}\right)=1$, and holonomy $\left.\operatorname{Spin}(7)\right)$, see [16]. Delete $k$ open disks $D_{1}^{m}, \ldots, D_{k}^{m} \subset J^{8}$ to obtain a manifold $M$, with $\partial M=S^{7} \times\langle k\rangle$. Let $g_{0}$ be the standard metric on $S^{7}$. Then clearly the metric $g_{0} \times\langle k\rangle$ on the boundary $S^{7} \times\langle k\rangle$ cannot be extended to a psc-metric on $M$ since otherwise one would construct a psc-metric on $J^{8}$. However, there are so called "exotic" metrics on $S^{7}$ which are not in the same connective component as the standard metric. Nevertheless, as we shall see, there is no any psc-metric $g^{\prime}$ on $S^{7}$, so that the metric $g^{\prime} \times\langle k\rangle$ could be extended to a psc-metric on $M$.

(5) Let $P=S^{1}$ with nontrivial Spin structure, so that $[P]$ is a generator of the cobordism group $\Omega_{1}^{\text {Spin }}=\mathbf{Z} / 2$.

Let $d \theta^{2}$ be the standard metric on the circle. The analysis of the ring structure of $\Omega_{*}^{\text {Spin }}$ shows that there exist many examples of simply connected manifolds $X$ which are not Spin cobordant to zero, however, the products $X \times P$ are, say $\partial Y=X \times P$.

Again, in general situation there is no obvious clue whether for some psc-metric $g_{X}$ on $X$ the product metric $g_{X}+d \theta^{2}$ on $X \times P$ could be extended to a psc-metric on $Y$ or not.

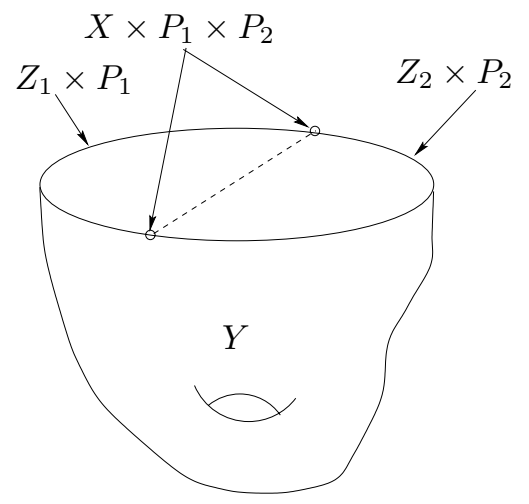

Figure 2

Now let $\left(P_{1}, g_{1}\right),\left(P_{2}, g_{2}\right)$ be two closed Riemannian manifolds, again, the metrics $g_{1}, g_{2}$ are not assumed to be of positive scalar curvature. Let $X$ be a closed manifold such that

- the product $X \times P_{1}$ is a boundary of a manifold $Z_{2}$, 
- the product $X \times P_{2}$ is a boundary of a manifold $Z_{1}$,

- the manifold $Z=Z_{1} \times P_{1} \cup \epsilon Z_{2} \times P_{2}$ is a boundary of a manifold $Y$ (where $\epsilon$ is an appropriate sign if the manifolds are oriented), see Figure 2.

Naive Question 2 Does there exist a psc-metric $g_{X}$ on $X$, so that

(a) the product metric $g_{X} \times g_{1}$ on $X \times P_{1}$ could be extended to a psc-metric $g_{Z_{2}}$ on $Z_{2}$,

(b) the product metric $g_{X} \times g_{2}$ on $X \times P_{1}$ could be extended to a psc-metric on $g_{Z_{1}} Z_{1}$,

(c) the metric $g_{Z_{1}} \times g_{P_{1}} \cup g_{Z_{2}} \times g_{P_{2}}$ on the manifold $Z=Z_{1} \times P_{1} \cup \epsilon Z_{2} \times P_{2}$ could be extended to a psc-metric $g_{Y}$ on $Y$ ?

1.2 Manifolds with singularities Perhaps, one can recognize that the above naive questions are actually about the existence of a psc-metric on a manifold with the Baas-Sullivan singularities, see [28], [2]. In particular, a $\mathbf{Z} / k_{-}$ manifold $M$ is a manifold with boundary $\partial M$ diffeomorphic to the product $\beta M \times\langle k\rangle$. Then a metric $g$ on $M$ is a regular Riemannian metric on $M$ such that it is product metric near the boundary, and its restriction on each two components $\beta M \times\{i\}, \beta M \times\{j\}$ are isometric via the above diffeomorphism. To get the singularity one has to identify the components $\beta M \times\{i\}$ with a single copy of $\beta M$. Similarly a Riemannian metric may be defined for the case of general singularities. We give details in Section 7 .

Thus manifolds with the Baas-Sullivan singularities provide an adequate environment to reformulate the above naive question. Let $\Sigma=\left(P_{1}, \ldots, P_{q}\right)$ be a collection of closed manifolds, and $M$ be a $\Sigma$-manifold (or manifold with singularities of the type $\Sigma$ ), see [2], [19], [3] for definitions. For example, if $\Sigma=(P)$, where $P=\langle k\rangle$, a $\Sigma$-manifold $M$ is $\mathbf{Z} / k$-manifold. Then the above questions lead to the following one:

Question Under which conditions does a $\Sigma$-manifold $M$ admit a psc-metric?

Probably it is hard to claim anything useful for a manifold with arbitrary singularities. We restrict our attention to Spin simply connected manifolds and very particular singularities. Now we introduce necessary notation.

Let $\Omega_{*}^{\text {Spin }}(\cdot)$ be the Spin-cobordism theory, and MSpin be the Thom spectrum classifying this theory. Let $\Omega_{*}^{\text {Spin }}(p t)=\Omega_{*}^{\text {Spin }}$ be the coefficient ring. Let $P_{1}=\langle 2\rangle=\{$ two points $\}, P_{2}$ be a circle with a nontrivial Spin structure, so that $\left[P_{2}\right]=\eta \in \Omega_{1}^{\text {Spin }} \cong \mathbf{Z} / 2$, and $P_{3},\left[P_{3}\right] \in \Omega_{8}^{\text {Spin }}$, is a Bott manifold, 
ie, a simply-connected manifold such that $\widehat{A}\left(P_{3}\right)=1$. There are different representatives of the Bott manifold $P_{3}$. Perhaps, the best choice is the Joyce manifold $J^{8}$, [16]. Let $\Sigma_{1}=\left(P_{1}\right), \Sigma_{2}=\left(P_{1}, P_{2}\right), \Sigma_{3}=\left(P_{1}, P_{2}, P_{3}\right)$, and $\eta=\left(P_{2}\right)$. We denote by $\Omega_{*}^{\text {Spin }, \Sigma_{i}}(\cdot)$ the cobordism theory of Spin-manifolds with $\Sigma_{i}$-singularities, and by $M \operatorname{Spin}^{\Sigma_{i}}$ the spectra classifying these theories, $i=1,2,3$. We also study the theory $\Omega_{*}^{S p i n, \eta}(\cdot)$, and the classifying spectrum for this theory is denoted as $M \operatorname{Spin}^{\eta}$. We use notation $\Sigma$ for the above singularities $\Sigma_{1}, \Sigma_{2}, \Sigma_{3}$ or $\eta$.

Let $K O_{*}(\cdot)$ be the periodic real $K$-theory, and $K O$ be the classifying $\Omega-$ spectrum. The Atiyah-Bott-Shapiro homomorphism $\alpha: \Omega_{*}^{S p i n} \longrightarrow K O_{*}$ induces the map of spectra

$$
\alpha: M \operatorname{Spin} \longrightarrow K O \text {. }
$$

It turns out that for our choice of singularities $\Sigma$ the spectrum $M S_{\text {pin }}{ }^{\Sigma}$ splits as a smash product $M \operatorname{Spin}^{\Sigma}=M \operatorname{Spin} \wedge X_{\Sigma}$ for some spectra $X_{\Sigma}$ (see Theorems $3.1,6.1)$. We would like to introduce the real $K$-theories $K O_{*}^{\Sigma}(\cdot)$ with the singularities $\Sigma$. We define the classifying spectrum for $K O_{*}^{\Sigma}(\cdot)$ by $K O^{\Sigma}=$ $K O \wedge X_{\Sigma}$. The $K$-theories $K O_{*}^{\Sigma}(\cdot)$ may be identified with the well-known $K$-theories. Indeed,

$$
K O_{*}^{\Sigma_{1}}(\cdot)=K O_{*}(\cdot ; \mathbf{Z} / 2), \quad K O_{*}^{\eta}(\cdot)=K_{*}(\cdot), \quad K O_{*}^{\Sigma_{2}}(\cdot)=K_{*}(\cdot ; \mathbf{Z} / 2),
$$

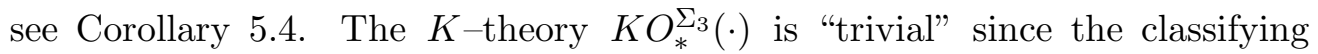
spectrum $\mathrm{KO}^{\Sigma_{3}}$ is contractible, see Corollary 6.4. Now the map $\alpha$ from (1) induces the map

$$
\alpha^{\Sigma}: M \operatorname{Spin}^{\Sigma}=M \operatorname{Spin} \wedge X_{\Sigma} \stackrel{\alpha \wedge 1}{\longrightarrow} K O \wedge X_{\Sigma}=K O^{\Sigma}
$$

and the homomorphism of the coefficient rings

$$
\alpha^{\Sigma}: \Omega_{*}^{S p i n, \Sigma} \longrightarrow K O_{*}^{\Sigma} \text {. }
$$

We define the integer $d(\Sigma)$ as follows:

$$
d\left(\Sigma_{1}\right)=6, \quad d\left(\Sigma_{2}\right)=8, \quad d\left(\Sigma_{3}\right)=17, \quad d(\eta)=7 .
$$

Recall that if $M$ is a $\Sigma$-manifold, then (depending on the length of $\Sigma$ ), the manifolds $\beta_{i} M, \beta_{i j} M, \beta_{i j k} M$ (as $\Sigma$-manifolds) are defined in canonical way. In particular, for $\Sigma=\Sigma_{1}, \eta$, there is a manifold $\beta_{i} M$ such that $\partial M=\beta_{i} M \times P_{i}$, for $\Sigma=\Sigma_{2}$, there are $\Sigma$-manifolds $\beta_{1} M, \beta_{2} M, \beta_{12} M$, and for $\Sigma=\Sigma_{3}$ there are $\Sigma$-manifolds $\beta_{i} M, \beta_{i j} M, \beta_{i j k} M$. These manifolds may be empty. The manifolds $\beta_{i} M, \beta_{i j} M$ and $\beta_{i j k} M$ are called $\Sigma$-strata of $M$.

We say that a $\Sigma$-manifold $M$ is simply connected if $M$ itself is simply connected and all $\Sigma$-strata of $M$ are simply connected manifolds. 
1.3 Main geometric result The following theorem is the main geometric result of this paper.

Theorem 1.1 Let $M^{n}$ be a simply connected Spin $\Sigma$-manifold of dimension $n \geq d(\Sigma)$, so that all $\Sigma$-strata manifolds are nonempty manifolds. Then $M$ admits a metric of positive scalar curvature if and only if $\alpha^{\Sigma}([M])=0$ in the group $K O_{n}^{\Sigma}$.

We complete the proof of Theorem 1.1 only at the end of the paper. However, we would like to present here the overview of the main ingredients of the proof.

1.4 Key ideas and constructions of the proof There are two parts of Theorem 1.1 to prove. The first "if" part is almost "pure topological". The second "only if" part has more analytical flavor. We start with the topological ingredients.

The first key construction which allows to reduce the question on the existence of a psc-metric to a topological problem, is the Surgery Lemma. This fundamental observation originally is due to Gromov-Lawson [9] and Schoen-Yau [27]. We generalize the Surgery Lemma for simply connected Spin $\Sigma$-manifolds.

This generalization is almost straightforward, however we have to describe the surgery procedure for $\Sigma-$ manifolds.

To explain the difference with the case of regular surgery, we consider the example when $M$ is a $\mathbf{Z} / k$-manifold, ie, $\partial M=\beta M \times\langle k\rangle$. There are two types of surgeries here. The first one is to do surgery on the interior of $M$, and the second one is to do surgery on each manifold $\beta M$.



Figure 3: The manifold $M^{\prime}$

We start with the second one. Let $M$ be a $\mathbf{Z} / k$-manifold, with a psc-metric $g_{M}$. We have $\partial M=\beta M \times\langle k\rangle$, where $g_{\beta M}$ is a psc-metric. Let $S^{p} \times D^{n-p-1} \subset \beta M$, and $V$ be a trace of the surgery along the sphere $S^{p}$, ie, $\partial V=-\beta M \cup \beta^{\prime} M$. We assume that $n-p-1 \geq 3$, so we can use the regular Surgery Lemma to push a psc-metric through the manifold $V$ to obtain a psc-metric $g_{V}$ which is a product near the boundary. Then we attach $k$ copies of $V$ to obtain a manifold $M^{\prime}=M \cup_{\partial M} V \times\langle k\rangle$, see Figure 3. Clearly the metrics $g_{M}$ and $g_{V}$ match along a color of the common boundary, giving a psc-metric $g^{\prime}$ on $M^{\prime}$. 
The first type of surgery is standard. Let $S^{\ell} \times D^{n-\ell} \subset M$ be a sphere together with a tubular neighborhood inside the interior of the manifold $M$. Denote by $M^{\prime \prime}$ the result of surgery on $M$ along the sphere $S^{\ell}$. Notice that $\partial M^{\prime \prime}=\partial M$. Then again the regular Surgery Lemma delivers a psc-metric on $M^{\prime \prime}$.

The case of two and more singularities requires a bit more care. We discuss the general Surgery procedure for $\Sigma$-manifolds in Section 7 . The Bordism Theorem (Theorem 7.3) for simply connected $\Sigma$-manifolds reduces the existence question of a positive scalar curvature to finding a $\Sigma$-manifold within the cobordism class $[M]_{\Sigma}$ equipped with a psc-metric.

To solve this problem we use the ideas and results due to S Stolz [29], [30]. The magic phenomenon discovered by S Stolz is the following. Let us start with the quaternionic projective space $\mathbf{H P}^{2}$ equipped with the standard metric $g_{0}$ (of constant positive curvature). It is not difficult to see that the Lie group

$$
G=P S p(3)=S p(3) / \text { Center, }
$$

acts by isometries of the metric $g_{0}$ on $\mathbf{H P}^{2}$. Here Center $\cong \mathbf{Z} / 2$ is the center of the group $S p(3)$. Then given a smooth bundle $E \stackrel{p}{\longrightarrow} B$ of compact Spinmanifolds, with a fiber $\mathbf{H P}^{2}$, and a structure group $G$, there is a straightforward construction of a psc-metric on the manifold $E$, the total space of this bundle. (A bundle with the above properties is called a geometric $\mathbf{H} \mathbf{P}^{2}$-bundle.) The construction goes as follows. One picks an arbitrary metric $g_{B}$ on a manifold $B$. Then locally, over an open set $U \subset B$, a metric on $p^{-1}(U) \cong U \times \mathbf{H P}^{2}$ is given as product metric $\left.g_{E}\right|_{p^{-1}(U)}=\left.g_{B}\right|_{U} \times g_{0}$. By scaling the metric $g_{0}$, one obtains that the scalar curvature of the metric $\left.g_{E}\right|_{p^{-1}(U)}$ is positive. Since the structure group of the bundle acts by isometries of the metric $g_{0}$, one easily constructs a psc-metric $g_{E}$ on $E$.

Perhaps, this general construction was known for ages. The amazing feature of geometric $\mathbf{H P}^{2}$-bundles is that their total spaces, the manifolds $E$, generate the kernel of the Atiyah-Bott-Shapiro transformation $\alpha: \Omega_{n}^{\text {Spin }} \longrightarrow K O_{n}$. In more detail, given an $\mathbf{H P}^{2}$-bundle $E^{n} \stackrel{p}{\longrightarrow} B^{n-8}$, there is a classifying map $f: B^{n-8} \longrightarrow B G$ which defines a cobordism class $[(B, f)] \in \Omega_{n-8}^{S p i n}(B G)$. The correspondence $[(B, f)] \mapsto[E] \in \Omega_{n}^{\text {Spin }}$ defines the transfer map

$$
T: \Omega_{n-8}^{\text {Spin }}(B G) \longrightarrow \Omega_{n}^{\text {Spin }} .
$$

Stolz proves [29] that $\operatorname{Im} T=\operatorname{Ker} \alpha$. Thus the manifolds $E$ deliver representatives in each cobordism class of the kernel Ker $\alpha$.

We adopt this construction for manifolds with singularities. First we notice that if a geometric $\mathbf{H P}^{2}$-bundle $E \stackrel{p}{\longrightarrow} B$ is such that $B$ is a $\Sigma$-manifold, 
then $E$ is also a $\Sigma$-manifold. In particular we obtain the induced transfer map

$$
T^{\Sigma}: \Omega_{*}^{\text {Spin }, \Sigma}(B G) \longrightarrow \Omega_{*+8}^{\text {Spin, }, \Sigma} .
$$

The key here is to prove that $\operatorname{Im} T^{\Sigma}=\operatorname{Ker} \alpha^{\Sigma}$. This requires complete information on the homotopy type of the spectra $M \operatorname{Spin}^{\Sigma}$. Sections 3-6 are devoted to study of the spectra $M \operatorname{Spin}^{\Sigma}$.

The second part, the proof of the "only if" statement, is geometric and analytic by its nature. We explain the main issues here for the case of $\mathbf{Z} / k$-manifolds. Recall that for a Spin manifold $M$ the direct image $\alpha([M]) \in K O_{n}$ is nothing else but the topological index of $M$ which coincides (via the Atiyah-Singer index theorem) with the analytical index $\operatorname{ind}(M) \in K O_{n}$ of the corresponding Dirac operator on $M$. Then the Lichnerowicz formula and its modern versions imply that the analytical index $\operatorname{ind}(M)$ vanishes if there is a psc-metric on $M$.

Thus if we would like to give a similar line of arguments for $\mathbf{Z} / k$-manifolds, we face the following issues. To begin with, we should have the Dirac operator to be well-defined on a $\operatorname{Spin} \mathbf{Z} / k$-manifold. Then we have to define the $\mathbf{Z} / k$-version of the analytical index $\operatorname{ind}_{\mathbf{z} / k}(M) \in K O_{n}^{\langle k\rangle}$ and to prove the vanishing result, ie, that $\operatorname{ind}_{\mathbf{Z} / k}(M)=0$ provided that there is a psc-metric on $M$. Thirdly we must identify the analytical index $\operatorname{ind}_{\mathbf{Z} / k}(M)$ with the direct image $\alpha^{\langle k\rangle}([M]) \in K O_{n}^{\langle k\rangle}$, ie, to prove the $\mathbf{Z} / k-\bmod$ version of the index theorem. These issues were already addressed, and, in the case of $S_{p i n}{ }^{c}$-manifolds, resolved by Freed [5], [6], Freed \& Melrose [7], Higson [11], Kaminker \& Wojciechowski [14], and Zhang [34, 35]. Unfortunately, the above papers study mostly the case of $\operatorname{Spin}^{c} \mathbf{Z} / k$-manifolds (with the exception of [34, 35] where the mod 2 index is considered), and the general case of $\operatorname{Spin} \mathbf{Z} / k$-manifolds is essentially left out in the cited work. The paper [22] by J. Rosenberg shows that the Dirac operator and its index are well-defined for $\mathbf{Z} / k$-manifolds and there the index vanishes if a Spin $\mathbf{Z} / k$-manifold has psc-metric. The case of general singularities $\Sigma$ require more work. Here we use the results of [22] to prove that if a $\Sigma$-manifold $M$ has a psc-metric, then $\alpha^{\Sigma}([M])=0$ in the group $K O^{\Sigma}$. In order to prove this fact we essentially use the specific homotopy features of the spectra $M \operatorname{Spin}^{\Sigma}$.

The plan is the following. We give necessary definitions and constructions on manifolds with singularities in Section 2. The next four sections are devoted to homotopy-theoretical study of the spectra $\operatorname{MSpin}^{\Sigma}$. We describe the homotopy type of the spectra $M \operatorname{Spin}^{\Sigma_{1}}, M_{S_{p i n}{ }^{\Sigma_{2}}}$, and $M \operatorname{Spin}^{\eta}$ in Section 3. We describe a product structure of these spectra in Section 4. In Section 5 we describe a splitting of the spectra $M \operatorname{Spin}^{\Sigma}$ into indecomposable spectra. In 
Section 6 we describe the homotopy type of the spectrum $M \operatorname{Spin}^{\Sigma_{3}}$. We prove the Surgery Lemma for manifolds with singularities in Section 7. Section 8 is devoted to the proof of Theorem 1.1.

It is a pleasure to thank Hal Sadofsky for helpful discussions on the homotopy theory involved in this paper, and acknowledge my appreciation to Stephan Stolz for numerous discussions about the positive scalar curvature. The author also would like to thank the Department of Mathematics of the National University of Singapore for hospitality (this was Fall of 1999). The author is thankful to Jonathan Rosenberg for his interest to this work and useful discussions. Finally, the author thanks the referee for helpful suggestions.

\section{Manifolds with singularities}

Here we briefly recall basic definitions concerning manifolds with the BaasSullivan singularities. Let $G$ be a stable Lie group. We will be interested in the case when $G=\operatorname{Spin}$. Consider the category of smooth compact manifolds with a stable $G$-structure in their stable normal bundle.

2.1 General definition Let $\Sigma=\left(P_{1}, \ldots, P_{k}\right)$, where $P_{1}, \ldots, P_{k}$ are arbitrary closed manifolds (possibly empty). It is convenient to denote $P_{0}=p t$. Let $I=\left\{i_{1}, \ldots, i_{q}\right\} \subset\{0,1, \ldots, k\}$. We denote $P^{I}=P_{i_{1}} \times \ldots \times P_{i_{q}}$.

Definition 2.1 We call a manifold $M$ a $\Sigma$-manifold if there are given the following:

(i) a partition $\partial M=\partial_{0} M \cup \partial_{1} M \cup \ldots \cup \partial_{k} M$ of its boundary $\partial M$ such that the intersection $\partial_{I} M=\partial_{i_{1}} M \cap \ldots \cap \partial_{i_{q}} M$ is a manifold for every collection $I=\left\{i_{1}, \ldots, i_{q}\right\} \subset\{0,1, \ldots, k\}$, and its boundary is equal to

$$
\partial\left(\partial_{I} M\right)=\bigcup_{j \notin I}\left(\partial_{I} M \cap \partial_{j} M\right) ;
$$

(ii) compatible product structures (ie, diffeomorphisms preserving the stable $G$-structure)

$$
\phi_{I}: \partial_{I} M \longrightarrow \beta_{I} M \times P^{I} \text {. }
$$

Compatibility means that if $I \subset J$ and $\iota: \partial_{J} M \longrightarrow \partial_{I} M$ is the inclusion, then the map

$$
\phi_{I} \circ \iota \circ \phi_{J}^{-1}: \beta_{J} M \times P^{J} \longrightarrow \beta_{I} M \times P^{I}
$$

is identical on the direct factor $P^{I}$. 
To get actual singularities we do the following. Two points $x, y$ of a $\Sigma$-manifold $M$ are equivalent if they belong to the same manifold $\partial_{I} M$ for some $I \subset$ $\{0,1, \ldots, k\}$ and $p r \circ \phi_{I}(x)=p r \circ \phi_{J}(y)$, where $p r: \beta_{I} M \times P^{I} \longrightarrow \beta_{I} M$ is the projection on the direct factor. The factor-space of $M$ under this equivalence relation is called the model of the $\Sigma$-manifold $M$ and is denoted by $M_{\Sigma}$. Actually it is convenient to deal with $\Sigma$-manifolds without considering their models. Indeed, we only have to make sure that all constructions are consistent with the projections $\pi: M \longrightarrow M_{\Sigma}$. The boundary $\delta M$ of a $\Sigma$-manifold $M$ is the manifold $\partial_{0} M$. If $\delta M=\emptyset$, we call $M$ a closed $\Sigma$-manifold. The boundary $\delta M$ is also a $\Sigma$-manifold with the inherited decomposition $\partial_{I}(\delta M)=$ $\partial_{I} M \cap \delta M$. The manifolds $\beta_{I} M$ also inherit a structure of a $\Sigma$-manifold:

$$
\partial_{j}\left(\beta_{I} M\right)=\left\{\begin{array}{cl}
\emptyset & \text { if } j \in I, \\
\beta_{\{j\} \cup I} M \times P_{j} & \text { otherwise. }
\end{array}\right.
$$

Here we denote $\beta_{I} M=\beta_{i_{1}}\left(\beta_{i_{2}}\left(\cdots \beta_{i_{q}} M\right) \cdots\right)$ for $I=\left\{i_{1}, \ldots, i_{q}\right\} \subset\{1, \ldots, k\}$. Let $(X, Y)$ be a pair of spaces, and $f:(M, \delta M) \longrightarrow(X, Y)$ be a map. Then the pair $(M, f)$ is a singular $\Sigma$-manifold of $(X, Y)$ if the map $f$ is such that for every index subset $I=\left\{i_{1}, \ldots, i_{q}\right\} \subset\{1, \ldots, k\}$ the map $\left.f\right|_{\partial_{I} M}$ is decomposed as $\left.f\right|_{\partial_{I} M}=f_{I} \circ p r \circ \phi_{I}$, where the map $\phi_{I}$ as above, pr: $\beta_{I} M \times P^{I} \longrightarrow \beta_{I} M$ is the projection on the direct factor, and $f_{I}: \beta_{I} M \longrightarrow X$ is a continuous map. The maps $f_{I}$ should be compatible for different indices $I$ in the obvious sense.

Remark 2.2 Let $(M, f)$ be a singular $\Sigma$-manifold, then the map $f$ factors through as $f=f_{\Sigma} \circ \pi$, where $\pi: M \longrightarrow M_{\Sigma}$ is the canonical projection, and $f_{\Sigma}: M_{\Sigma} \longrightarrow X$ is a continuous map. We also notice that singular $\Sigma$-manifolds may be identified with their $\Sigma$-models.

The cobordism theory $\Omega_{*}^{G, \Sigma}(\cdot)$ of $\Sigma$-manifolds is defined in the standard way. In the case of interest, when $G=\operatorname{Spin}$, we denote $M \operatorname{Spin}^{\Sigma}$ a spectrum classifying the cobordism theory $\Omega_{*}^{\operatorname{Spin}, \Sigma}(\cdot)$.

2.2 The case of two and three singularities We start with the case $\Sigma=\left(P_{1}, P_{2}\right)$. Then if $M$ is a $\Sigma$-manifold, we have that the diffeomorphisms

$$
\begin{gathered}
\phi: \partial M \stackrel{\cong}{\longrightarrow} \partial_{1} M \cup \partial_{2} M, \\
\phi_{i}: \partial_{i} M \stackrel{\cong}{\longrightarrow} \beta_{i} M \times P_{i}, \quad i=1,2 ; \\
\phi_{12}: \partial_{1} M \cap \partial_{2} M \stackrel{\cong}{\longrightarrow} \beta_{12} M \times P_{1} \times P_{2}
\end{gathered}
$$

are given. We always assume that the manifold $\beta_{12} M \times P_{1} \times P_{2}$ is embedded into $\partial_{1} M$ and $\partial_{2} M$ together with a color: 


$$
\beta_{12} M \times P_{1} \times P_{2} \times I \subset \partial_{1} M, \partial_{2} M .
$$

Thus we actually have the following decomposition of the boundary $\partial M$ :

$$
\partial M \cong \partial_{1} M \cup\left(\beta_{12} M \times P_{1} \times P_{2} \times I\right) \cup \partial_{2} M,
$$

so the manifold $\beta_{12} M \times P_{1} \times P_{2}$ is "fattened" inside $\partial M$. Also we assume that the boundary $\partial M$ is embedded into $M$ together with a color $\partial M \times I \subset M$, see Figure 4 .



Figure 4

The case when $\Sigma=\left(P_{1}, P_{2}, P_{3}\right)$ is the most complicated one we are going to work with.

Let $M$ be a closed $\Sigma$-manifold, then we $\quad \beta_{123} M \times P_{1} \times P_{2} \times P_{3}$ are given the diffeomorphisms:

$\phi: \partial M \stackrel{\cong}{\longrightarrow} \partial_{1} M \cup \partial_{2} M \cup \partial_{3} M$,

$\phi_{i}: \partial_{i} M \stackrel{\cong}{\longrightarrow} \beta_{i} M \times P_{i}, \quad i=1,2,3 ;$

$\phi_{i j}: \partial_{i} M \cap \partial_{j} M \stackrel{\cong}{\longrightarrow} \beta_{i j} M \times P_{i} \times P_{j}$,

$\phi_{123}: \partial_{1} M \cap \partial_{2} M \cap \partial_{3} M \stackrel{\cong}{\longrightarrow}$

$$
\beta_{123} M \times P_{1} \times P_{2} \times P_{3}
$$

where $i, j=1,2,3, \quad i \neq j$, see Figure 5 .

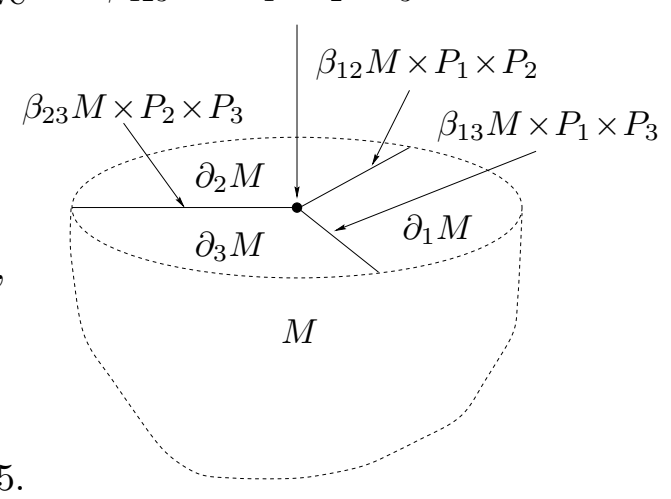

Figure 5

First, we assume here that the boundary $\partial M$ is embedded into $M$ together with a color $(0,1] \times \partial M$. The decomposition

$$
\partial M \stackrel{\phi}{\longrightarrow} \partial_{1} M \cup \partial_{2} M \cup \partial_{3} M
$$

gives also the "color" structure on $\partial M$.

We assume that the boundary $\partial\left(\partial_{i} M\right)$ is embedded into $\partial_{i} M$ together with the color $(0,1] \times \partial\left(\partial_{i} M\right)$.

Even more, we assume that the manifold $\beta_{123} M \times P_{1} \times P_{2} \times P_{3}$ is embedded into the boundary $\partial M$ together with its normal tube:

$$
\beta_{123} M \times P_{1} \times P_{2} \times P_{3} \times D^{2} \subset \partial M,
$$


so that the colors of the manifolds $\beta_{23} M \times P_{2} \times P_{3} \times I \quad \beta_{123} M \times P_{1} \times P_{2} \times P_{3} \times D^{2}$

$$
\beta_{i j} M \times P_{i} \times P_{j} \subset \partial_{i} M \cap \partial_{j} M
$$

are compatible with this embedding, as is shown on Figure 6. As in the case of two singularities, the submanifolds

$$
\begin{aligned}
& \beta_{i j} M \times P_{i} \times P_{j} \text { and } \\
& \beta_{123} M \times P_{1} \times P_{2} \times P_{3}
\end{aligned}
$$

are "fattened" inside the boundary $\partial M$. Furthermore, we assume that there are not any corners in the above color decomposition.

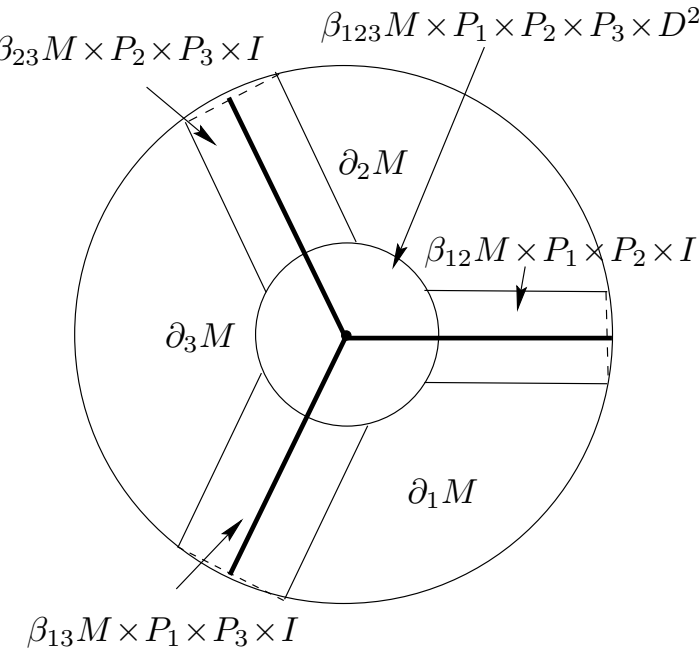

Figure 6

2.3 Bockstein-Sullivan exact sequence Let $M G$ be the Thom spectrum classifying the cobordism theory $\Omega_{*}^{G}(\cdots)$. Let $\Sigma=(P)$, and $p=\operatorname{dim} P$. Then there is a stable map $S^{p} \stackrel{[P]}{\longrightarrow} M G$ representing the element $[P]$. Then we have the composition

$$
\cdot[P]: \Sigma^{p} M G=S^{p} \wedge M G \stackrel{[P] \wedge I d}{\longrightarrow} M G \wedge M G \stackrel{\mu}{\longrightarrow} M G
$$

where $\mu$ is the map giving $M G$ a structure of a ring spectrum. Then the cofiber, the spectrum $M G^{\Sigma}$ of the map

$$
\Sigma^{p} M G \stackrel{\cdot[P]}{\longrightarrow} M G \stackrel{\pi}{\longrightarrow} M G^{\Sigma}
$$

is a classifying spectrum for the cobordism theory $\Omega_{*}^{G, \Sigma}$. The cofiber (4) induce the long exact Bockstein-Sullivan sequence

$$
\cdots \rightarrow \Omega_{n-p}^{G}(X, A) \stackrel{\cdot[P]}{\longrightarrow} \Omega^{G}(X, A) \stackrel{\pi}{\longrightarrow} \Omega_{n-p}^{G, \Sigma}(X, A) \stackrel{\beta}{\longrightarrow} \Omega_{n-p-1}^{G}(X, A) \rightarrow \cdots
$$

for any $C W$-pair $(X, A)$. Similarly, if $\Sigma_{j}=\left(P_{1}, \ldots, P_{j}\right), j=1, \ldots, k$, then there is a cofiber

$$
\Sigma^{p_{j}} M G^{\Sigma_{j-1}} \stackrel{\cdot\left[P_{j}\right]}{\longrightarrow} M G^{\Sigma_{j-1}} \stackrel{\pi_{j}}{\longrightarrow} M G^{\Sigma_{j}}
$$

induce the exact Bockstein-Sullivan sequence

$$
\cdots \stackrel{\beta_{j}}{\longrightarrow} \Omega_{n-p_{j}}^{G, \Sigma_{j-1}}(X, A) \stackrel{\cdot\left[P_{j}\right]}{\longrightarrow} \Omega_{n}^{G, \Sigma_{j-1}}(X, A) \stackrel{\pi_{j}}{\longrightarrow} \Omega_{n}^{G, \Sigma_{j}}(X, A) \stackrel{\beta_{j}}{\longrightarrow} \cdots
$$

for any $C W$-pair $(X, A)$. We shall use the Bockstein-Sullivan exact sequences (5), (6) throughout the paper. 


\section{The spectra $M \operatorname{Spin}^{\Sigma_{1}}, M_{S p i n}^{\Sigma_{2}}$ and $M \operatorname{Spin}^{\eta}$}

Let $M(2)$ be the mod 2 Moore spectrum with the bottom cell in zero dimension, ie, $M(2)=\Sigma^{-1} \mathbf{R} \mathbf{P}^{2}$. We consider also the spectrum $\Sigma^{-2} \mathbf{C P}^{2}$ and the spectrum $Y=M(2) \wedge \Sigma^{-2} \mathbf{C P}^{2}$ which was first studied by M Mahowald, [17]. Here is the result on the spectra $M \operatorname{Spin}^{\Sigma_{1}}, \operatorname{MSpin}^{\Sigma_{2}}$ and $M \operatorname{Spin}^{\eta}$.

Theorem 3.1 There are homotopy equivalences:

(i) $\operatorname{MSpin}^{\Sigma_{1}} \cong \operatorname{MSpin} \wedge M(2)$,

(ii) $M \operatorname{Spin}^{\eta} \cong M \operatorname{Spin} \wedge \Sigma^{-2} \mathbf{C P}^{2}$,

(iii) $\operatorname{MSpin}^{\Sigma_{2}} \cong M \operatorname{Spin} \wedge Y$.

Proof Let $\iota: S^{0} \longrightarrow M S p i n$ be a unit map. The main reason why the above homotopy equivalences hold is that the elements $2, \eta \in \Omega_{*}^{\text {Spin }}$ are in the image of the homomorphism $\iota_{*}: S_{*}^{0} \longrightarrow \Omega_{*}^{\text {Spin }}$. Indeed, consider first the spectrum $M S_{\text {Pin }}^{\eta}$. Let $S^{1} \stackrel{\eta}{\longrightarrow} S^{0}$ be a map representing $\eta \in \pi_{1}\left(S^{0}\right)$. We obtain the cofibration:

$$
S^{1} \stackrel{\eta}{\longrightarrow} S^{0} \stackrel{\pi}{\longrightarrow} \Sigma^{-2} \mathbf{C P}^{2} .
$$

Then the composition $S^{1} \stackrel{\eta}{\longrightarrow} S^{0} \stackrel{\iota}{\longrightarrow} M$ Spin represents $\eta \in M$ Spin $_{1}$. Let $\cdot \eta$ be the map

$$
\cdot \eta: S^{1} \wedge M \operatorname{Spin} \stackrel{\iota \eta \wedge 1}{\longrightarrow} M \operatorname{Spin} \wedge M \operatorname{Spin} \stackrel{\mu}{\longrightarrow} M \operatorname{Spin},
$$

where $\mu$ is a multiplication. Note that the diagram

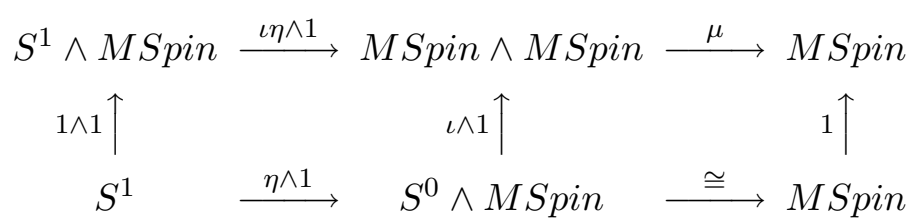

commutes since the map $\iota: S^{0} \longrightarrow$ MSpin represents a unit of the ring spectrum MSpin. We obtain a commutative diagram of cofibrations:

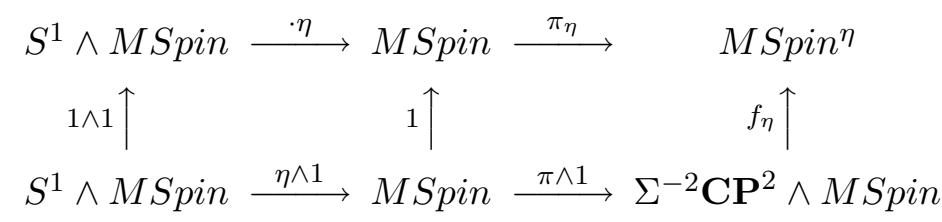

where $f_{\eta}: M$ Spin $^{\eta} \longrightarrow \Sigma^{-2} \mathbf{C P}^{2} \wedge M$ Spin $\cong M$ Spin $\wedge \Sigma^{-2} \mathbf{C P}^{2}$ gives a homotopy equivalence by 5 -lemma. The proof for the spectrum $M S_{p i n}{ }^{\Sigma_{1}}=$ $M \operatorname{Spin}^{\langle 2\rangle}$ is similar. 
Consider the spectrum $M \operatorname{Spin}^{\Sigma_{2}}$. First we note that the bordism theory $\Omega_{*}^{\text {Spin }, \Sigma_{2}}(\cdot)=\Omega_{*}^{\text {Spin },\left(P_{1}, P_{2}\right)}(\cdot)$ coincides with the theory $\Omega_{*}^{\text {Spin },\left(P_{2}, P_{1}\right)}(\cdot)$, where the order of singularities is switched. In particular, the spectrum $M \operatorname{Spin}^{\Sigma_{2}}$ is a cofiber in the following cofibration:

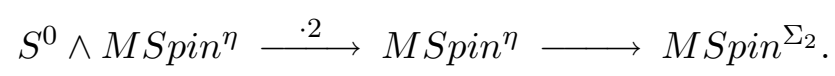

Here the map $\cdot 2: S^{0} \wedge M \operatorname{Spin}^{\eta} \longrightarrow M S p i n^{\eta}$ is defined as follows. Let $S^{0} \stackrel{2}{\longrightarrow} S^{0}$ be a map of degree 2 . Then the composition $S^{0} \stackrel{2}{\longrightarrow} S^{0} \stackrel{\iota}{\longrightarrow} M S p i n$ represents $2 \in \Omega_{0}^{\text {Spin }}$. The spectrum $M S p i n^{\eta}$ is a module (say, left) spectrum over MSpin, ie, there is a map $\mu_{L}^{\prime}: M \operatorname{Spin} \wedge M \operatorname{Spin}^{\eta} \longrightarrow M \operatorname{Spin}^{\eta}$ so that the diagram

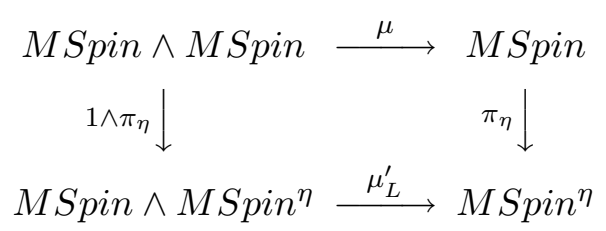

commutes. Then the map $\cdot 2$ is defined as composition:

$$
S^{0} \wedge M \operatorname{Spin}^{\eta} \stackrel{2 \iota \wedge 1}{\longrightarrow} M \operatorname{Spin} \wedge M \operatorname{Spin}^{\eta} \stackrel{\mu_{L}^{\prime}}{\longrightarrow} M \operatorname{Spin}^{\eta} .
$$

Note that the diagram

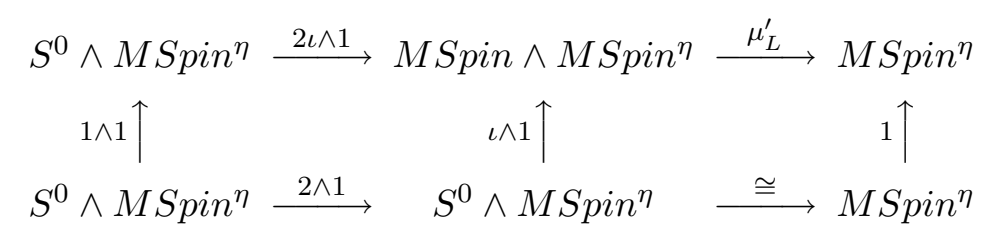

commutes since $S^{0} \stackrel{\iota}{\longrightarrow} M$ Spin represents a unit, and $M S p i n^{\eta}$ is a left module over the ring spectrum MSpin. We obtain the commutative diagram of cofibrations:

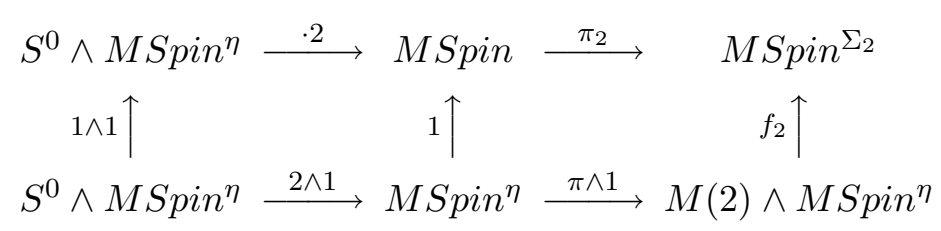

The map $f_{2}: M(2) \wedge M \operatorname{Spin}^{\eta} \longrightarrow M \operatorname{Spin}^{\Sigma_{2}}$ gives a desired homotopy equivalence. Thus we have $M \operatorname{Spin}^{\Sigma_{2}} \cong M(2) \wedge M \operatorname{Spin}^{\eta} \cong M \operatorname{Spin}^{\eta} \wedge M(2)=$ $M \operatorname{Spin} \wedge Y$.

Remark 3.2 In the above proof, we did not use any specific properties of the spectrum MSpin except that it is a ring spectrum. In fact, MSpin may be replaced by any other classic Thom spectrum. 
Later we prove that the homotopy equivalence

$$
M \operatorname{Spin}^{\Sigma_{3}} \sim M \operatorname{Spin} \wedge \Sigma^{-2} \mathbf{C P}^{2} \wedge V(1),
$$

where $V(1)$ is the cofiber of the Adams map $A: \Sigma^{8} M(2) \longrightarrow M(2)$. However, first we have to study the spectra $M \operatorname{Spin}^{\Sigma_{1}}, M_{S_{p i n}{ }^{\Sigma_{2}}}$ and $M \operatorname{Spin}^{\eta}$ in more detail.

\section{Product structure}

Recall that the spectrum MSpin is a ring spectrum. Here we work with the category of spectra, and commutativity of diagrams mean commutativity up to homotopy. Let, as above, $\iota: S^{0} \longrightarrow$ MSpin be the unit, and $\mu$ : MSpin $\wedge$ $M S p i n \longrightarrow M S p i n$ the map defining the product structure. Let $M \operatorname{Spin}^{\Sigma}$ be one of the spectrum we considered above. The natural map $\pi: M \operatorname{Spin} \longrightarrow$ $M \operatorname{Spin}^{\Sigma}$ turns the spectrum $M \operatorname{Spin}^{\Sigma}$ into a left and a right module over the spectrum MSpin, ie, there are maps

$$
\mu_{L}^{\prime}: M \operatorname{Spin} \wedge M \operatorname{Spin}^{\Sigma} \longrightarrow M \operatorname{Spin}^{\Sigma}, \mu_{R}^{\prime}: M \operatorname{Spin}^{\Sigma} \wedge M \operatorname{Spin} \longrightarrow M \operatorname{Spin}^{\Sigma},
$$

so that the diagrams

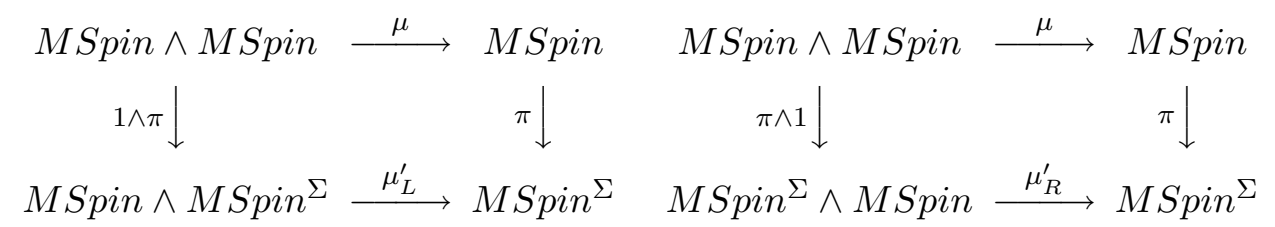

commute. We say that the spectrum $M \operatorname{Spin}^{\Sigma}$ has an admissible ring structure

$$
\mu^{\Sigma}: M \operatorname{Spin}^{\Sigma} \wedge M \operatorname{Spin}^{\Sigma} \longrightarrow M \operatorname{Spin}^{\Sigma}
$$

if the map $S^{0} \stackrel{\iota}{\longrightarrow} M \operatorname{Spin} \stackrel{\pi}{\longrightarrow} M \operatorname{Spin}^{\Sigma}$ is a unit, and the diagrams

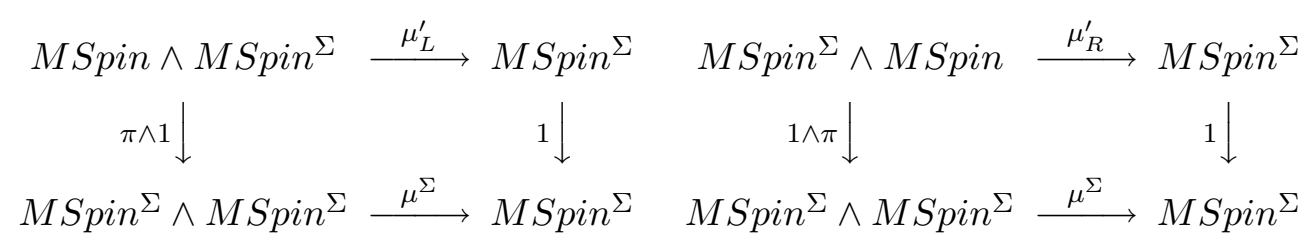

commute. The questions of existence, commutativity and associativity of an admissible product structure were thoroughly studied in [3], [19].

Theorem 4.1 (i) The spectrum $\operatorname{MSpin}^{\Sigma_{1}}$ does not admit an admissible product structure. 
(ii) The spectra $M \operatorname{Spin}^{\eta}, M \operatorname{Spin}^{\Sigma_{2}}$ and $\operatorname{MSpin}^{\Sigma_{3}}$ have admissible product structures $\mu^{\eta}, \mu^{\Sigma_{2}}=\mu^{(2)}$, and $\mu^{\Sigma_{3}}=\mu^{(3)}$ respectively.

(iii) For any choice of an admissible product structure $\mu^{\eta}$, it is commutative and associative. For any choice of admissible product structures $\mu^{(2)}$, and $\mu^{(3)}$, they are associative, but not commutative.

Proof Recall that for each singularity manifold $P_{i}$ there is an obstruction manifold $P_{i}^{\prime}$ with singularity. In the cases of interest, we have: $\left[P_{1}^{\prime}\right]_{\Sigma_{1}}=\eta \in$ $\Omega_{1}^{S p i n, \Sigma_{1}}$, which is non-trivial; and the obstruction $\left[P_{2}^{\prime}\right] \in \Omega_{3}^{S p i n, \Sigma_{2}}=0$, and $\left[P_{2}^{\prime}\right] \in \Omega_{3}^{S p i n, \eta}=0$. Thus [3, Lemma 2.2.1] implies that there is no admissible product structure in the cobordism theory $\Omega_{*}^{\text {Spin }, \Sigma_{1}}(\cdot)$, so the spectrum $\operatorname{MSpin}^{\Sigma_{1}}$ does not admit an admissible product structure. The obstruction element $\left[P_{3}^{\prime}\right]_{\Sigma_{3}} \in \Omega_{17}^{\operatorname{Spin}, \Sigma_{3}}$, and since $\operatorname{dim} P_{3}=8$ is even, the obstruction manifold $P_{3}^{\prime}$ is, in fact, a manifold without any singularities (see [19]), so the element $\left[P_{3}^{\prime}\right]_{\Sigma_{3}}$ is in the image $\operatorname{Im}\left(\Omega_{17}^{\text {Spin }} \longrightarrow \Omega_{17}^{\text {Spin }, \Sigma_{3}}\right)$. However, the elements of $\Omega_{17}^{\text {Spin }}$ are divisible by $\eta$, so they are zero in the group $\Omega_{17}^{\text {Spin, } \eta}$, and, consequently, in $\Omega_{17}^{\operatorname{Spin}, \Sigma_{3}}$.

The result of [3, Theorem 2.2.2] implies that the spectra $M \operatorname{Spin}^{\eta}, \operatorname{MSpin}^{\Sigma_{2}}$ and $\operatorname{MSpin}^{\Sigma_{3}}$ have admissible product structures $\mu^{(2)}$ and $\mu^{\eta}$ respectively.

It is also well-known [33] that the element $v_{1} \in \Omega_{2}^{S p i n, \Sigma_{2}}$ is an obstruction to the commutativity of the product structure $\mu^{(2)}$. An obstruction to the commutativity for the product structure $\mu^{\eta}$ lives in the group $\Omega_{5}^{\operatorname{Spin}, \Sigma_{2}}=0$. The obstructions to associativity are 3 -torsion elements, (see [3, Lemma 4.2.4]) so they all are zero.

\section{Homotopy structure of the spectra $M \operatorname{Spin}^{\Sigma}$}

First we recall the work of Anderson, Brown, and Peterson [1] on structure of the spectra MSpin, and of M Hopkins, M Hovey [13].

Let $K O_{*}(\cdot)$ be a periodic homological real $K$-theory, $K O$ be a corresponding $\Omega$-spectrum. Also let $k o$ be the connected cover of $K O$, and $k o\langle 2\rangle$ denote the 2 -connective cover of $k o$. It is convenient to identify the $2 n$-fold connective covers of the spectrum $K O$. Indeed, the $4 k$-fold connective cover of $K O$ is $\Sigma^{4 k} k o$ (when $k$ is even), and the $(4 k-2)$-fold connective cover is $\Sigma^{4 k-2} k o\langle 2\rangle$. Let $k u$ be a connected cover of the complex $K$-theory spectrum 
$K$. Let $\mathbf{H}(\mathbf{Z} / 2)$ denote the $\mathbf{Z} / 2$-Eilenberg-MacLane spectrum. Recall that $k o$ and $k u$ are the ring spectra with the coefficient rings:

$$
\begin{aligned}
& k o_{*} \cong \mathbf{Z}[\eta, \omega, b] /\left(2 \eta, \eta^{3}, \omega \eta, \omega^{2}-4 b\right), \quad \operatorname{deg} \eta=1, \operatorname{deg} \omega=4, \operatorname{deg} b=8 ; \\
& k u_{*} \cong \mathbf{Z}[v], \quad \operatorname{deg} v=2 .
\end{aligned}
$$

Let $I=\left(i_{1}, \ldots, i_{r}\right)$ be a partition (possibly empty) of $n=n(I)=\sum_{t=1}^{r} i_{t}$, $i_{t}>0$. Each partition $I$ defines a map $\pi^{I}: M S p i n \longrightarrow K O$ (which gives the $K O$-characteristic class, see [1]). If $I=\emptyset$ we denote $\pi^{\emptyset}$ by $\pi^{0}$, which coincides with the Atiyah-Bott-Shapiro orientation $\alpha: M \operatorname{Spin} \longrightarrow K O$.

Remark 5.1 Let $\mathcal{P}$ be a set of all partitions, which is an abelian group. We can make the set $\mathbf{Z}[\mathcal{P}]$ of linear combinations into a ring, where multiplication of partitions is defined by set union, and then to into a Hopf algebra with the diagonal $\Delta(I)=\sum_{I_{1}+I_{2}=I} I_{1} \otimes I_{2}$.

Let $\mu: M$ Spin $\wedge M$ Spin $\longrightarrow M$ Spin, $\mu^{\prime}: K O \wedge K O \longrightarrow K O$ denote the ring spectra multiplications. The Cartan formula says that

$$
\begin{array}{rlrl}
M S p i n & \wedge M \text { Spin } & \mu & \text { MSpin } \\
\sum\left(\pi^{I_{1}} \wedge \pi^{I_{2}}\right) & \downarrow \\
K O & \wedge K O \quad \stackrel{\mu^{\prime}}{\longrightarrow} \text { KO }
\end{array}
$$

Theorem 5.2 [1]

(1) Let $1 \notin I$. Then if $n(I)$ is even, the map $\pi^{I}: M$ Spin $\longrightarrow K O$ lifts to a map $\bar{\pi}^{I}: M S p i n \longrightarrow \Sigma^{4 n(I)} k o$. If $n(I)$ is odd, the map $\pi^{I}$ lifts to a map $\bar{\pi}^{I}: M \operatorname{Spin} \longrightarrow \Sigma^{4 n(I)-4} k o\langle 2\rangle$.

(2) There exist a countable collection $z_{k} \in H^{*}(M \operatorname{Spin} ; \mathbf{Z} / 2)$ such that the map

$$
\prod_{1 \notin I} \bar{\pi}^{I} \times \prod_{k} z_{k}: M S p i n \underset{\substack{1 \notin I, n(I) \text { even }}}{\longrightarrow} \sum_{\substack{1 \notin I, n(I) \text { odd }}} \Sigma^{4 n(I)} k o \times \sum^{4 n(I)-4} k o\langle 2\rangle \times \prod_{k} \Sigma^{\operatorname{deg} z_{k}} \mathbf{H}(\mathbf{Z} / 2)
$$

is a 2-local homotopy equivalence.

We use here the product symbol, however in the stable category of spectra the product and the coproduct, ie the wedge, are the same. We denote by $\rho^{I}$ the left inverses of the maps $\bar{\pi}^{I}$ (when $1 \notin I$ ). We denote also by $b$ an element in 
$\Omega_{8}^{S p i n}$ which is the image of the Bott element under the map $\rho^{0}$. The following Lemma due to M Hovey and M Hopkins [13]. Since some fragments of its proof will be used later, we provide an argument which essentially repeats [13].

Lemma 5.3 [13, Lemma 1] Let $I$ be a partition. Then $\pi^{I}(b)=0$ except for $\pi^{0}(b)=b$ and possibly $\pi^{1}(b) \in K O_{8}$ and $\pi^{1,1}(b) \in K O_{8}$. The elements $\pi^{1}(b)$, $\pi^{1,1}(b)$ are divisible by two in the group $\mathrm{KO}_{8}$. Further, the image of the Bott element $b$ is zero in $\mathrm{MO}_{8}$.

Proof In the case $1 \notin I, I \neq \emptyset$, the splitting shows that $\pi^{I}(b)=0$. The map $\pi^{I}:$ MSpin $\longrightarrow K O$ (for any partitions $I$ ) may be lifted to the $4 n(I)-$ connective cover of $K O$, as it is shown in [32]. Let $S^{0} \longrightarrow k o$ be a unit map, and $\rho^{0}: k o \longrightarrow M$ Spin be a left inverse of $\bar{\pi}^{0}$. The composition

$$
S^{0} \longrightarrow k o \stackrel{\rho^{0}}{\longrightarrow} M \operatorname{Spin} \stackrel{\pi^{I}}{\longrightarrow} \mathrm{KO}
$$

is null-homotopic for $I \neq \emptyset$. Let $\eta \in M \operatorname{Spin}_{1}=\mathbf{Z} / 2$ be a generator. It is well-known that the image of the map $S^{0} \longrightarrow$ MSpin on positive dimensional homotopy groups is $\left\{b^{n} \eta, b^{n} \eta^{2} \mid n \geq 0\right\}$. It implies that $\pi^{I} \rho^{0}\left(b^{n} \eta\right)=0$ and $\pi^{I} \rho^{0}\left(b^{n} \eta^{2}\right)=0$ for all partitions $I \neq \emptyset$. Since the unit map $S^{0} \longrightarrow M S p i n$ is a map of ring spectra, we have $\eta \cdot \pi^{I} \rho^{0}\left(b^{n}\right)=0$, so the elements $\pi^{I} \rho^{0}\left(b^{n}\right)$ are even for all partitions $I \neq \emptyset$. In particular, $\pi^{I}(b)$ is even for all $I \neq \emptyset$.

Let $p_{I}$ be the Pontryagin class corresponding to a partition $I$. Anderson, Brown and Peterson show that the Chern character ch $\left(\pi^{I}(x) \otimes \mathbf{C}\right)=p_{I}(x)+$ (higher terms), for $x \in \Omega_{\text {Spin }}^{*}(X)$. It implies that $p_{I}(b)$ are even elements for all $I \neq \emptyset$. The Pontryagin classes $p_{2}$ and $p_{1,1}=p_{1}^{2}$ determine the oriented cobordism ring $\Omega_{*}^{S O}$ in dimension 8 , so the Bott element goes to an even element in $\Omega_{8}^{S O}$ under the natural map MSpin $\longrightarrow M S O$. Thus the composition $M S p i n \longrightarrow M S O \longrightarrow M O$ takes the Bott element $b$ to zero.

We define the $K$-theory spectra with singularities $K O^{\Sigma_{1}}, K O^{\eta}$ and $K O^{\Sigma_{2}}$, as the cofibers:

$$
\begin{aligned}
& K O \wedge S^{0} \stackrel{1 \wedge 2}{\longrightarrow} K O \wedge S^{0} \stackrel{\pi}{\longrightarrow} K O \wedge M(2)=K O^{\Sigma_{1}} \\
& K O \wedge S^{1} \stackrel{1 \wedge \eta}{\longrightarrow} K O \wedge S^{0} \stackrel{\pi}{\longrightarrow} K O \wedge \Sigma^{-2} \mathbf{C P}^{2}=K O^{\eta} \\
& K O^{\eta} \wedge S^{0} \stackrel{1 \wedge 2}{\longrightarrow} K O^{\eta} \wedge S^{0} \stackrel{\pi}{\longrightarrow} K O^{\eta} \wedge M(2)=K O^{\Sigma_{2}}
\end{aligned}
$$

It is easy to derive (see, for example, [18]) the following statement. 
Corollary 5.4 The spectrum $K O^{\eta}$ is homotopy equivalent (as a ring spectrum) to the spectrum $K$, classifying the complex $K$-theory, and the spectrum $\mathrm{KO}^{\Sigma_{2}}$ is homotopy equivalent (as a ring spectrum) to the spectrum $K(1)$ classifying the first Morava $K$-theory.

We introduce also the notation:

$$
\begin{aligned}
& k o^{\Sigma_{1}}=k o \wedge M(2), \quad k o\langle 2\rangle^{\Sigma_{1}}=k o\langle 2\rangle \wedge M(2), \mathbf{H}(\mathbf{Z} / 2)^{\Sigma_{1}}=\mathbf{H}(\mathbf{Z} / 2) \wedge M(2) ; \\
& k o^{\eta}=k o \wedge \Sigma^{-2} \mathbf{C P}^{2}, k o\langle 2\rangle^{\eta}=k o\langle 2\rangle \wedge \Sigma^{-2} \mathbf{C P}^{2}, \mathbf{H}(\mathbf{Z} / 2)^{\eta}=\mathbf{H}(\mathbf{Z} / 2) \wedge \Sigma^{-2} \mathbf{C P}^{2} ; \\
& k o^{\Sigma_{2}}=k o^{\eta} \wedge M(2), k o\langle 2\rangle^{\Sigma_{2}}=k o\langle 2\rangle^{\eta} \wedge M(2), \mathbf{H}(\mathbf{Z} / 2)^{\Sigma_{2}}=\mathbf{H}(\mathbf{Z} / 2)^{\eta} \wedge M(2) .
\end{aligned}
$$

Let $I$ be a partition as above. The $K O$-characteristic numbers

$$
\pi^{I}: M \operatorname{Spin} \longrightarrow K O
$$

which are lifted to the connective cover $k o\langle 4 n(I)\rangle$ give the characteristic numbers

$$
\begin{array}{ll}
\pi_{\Sigma_{1}}^{I}=\pi^{I} \wedge 1: M \operatorname{Spin}^{\Sigma_{1}}=M \operatorname{Spin} \wedge M(2) \longrightarrow K O \wedge M(2) & =K O^{\Sigma_{1}}, \\
\pi_{\eta}^{I}=\pi^{I} \wedge 1: M \operatorname{Spin}^{\eta}=M \operatorname{Spin} \wedge \Sigma^{-2} \mathbf{C P}^{2} \longrightarrow K O \wedge \Sigma^{-2} \mathbf{C P}^{2} & =K O^{\eta}, \\
\pi_{\Sigma_{2}}^{I}=\pi^{I} \wedge 1: M \operatorname{Spin}^{\Sigma_{1}}=M \operatorname{Spin} \wedge Y \longrightarrow K O \wedge Y & =K O^{\Sigma_{2}},
\end{array}
$$

together with the lifts to the corresponding connective covers:

$$
\begin{aligned}
& \bar{\pi}_{\Sigma_{1}}^{I}=\bar{\pi}^{I} \wedge 1: \quad M \operatorname{Spin}^{\Sigma_{1}} \longrightarrow k o\langle 4 n(I)\rangle \wedge M(2) \quad=k o\langle 4 n(I)\rangle^{\Sigma_{1}} \\
& \bar{\pi}_{\eta}^{I}=\bar{\pi}^{I} \wedge 1: \quad M \operatorname{Spin}^{\eta} \longrightarrow k o\langle 4 n(I)\rangle \wedge \Sigma^{-2} \mathbf{C P}^{2}=k o\langle 4 n(I)\rangle^{\eta} \\
& \bar{\pi}_{\Sigma_{2}}^{I}=\bar{\pi}^{I} \wedge 1: \quad M \operatorname{Spin}^{\Sigma_{1}} \longrightarrow k o\langle 4 n(I)\rangle \wedge Y \quad=k o\langle 4 n(I)\rangle^{\Sigma_{2}}
\end{aligned}
$$

Now we would like to identify the spectra $k o^{\Sigma}, k o\langle 4 n(I)\rangle^{\Sigma}$ for $\Sigma=\Sigma_{2}$ or $\eta$ for those partitions $I, 1 \notin I$. It is enough to determine a homotopy type of the spectra $k o^{\Sigma}$ and $k o\langle 2\rangle^{\Sigma}$.

Let $\mathcal{A}(1)$ be a subalgebra of the Steenrod algebra $\mathcal{A}_{2}$ generated by $1, S q^{1}, S q^{2}$. The cohomology $H^{*}(k o)$ as a module over Steenrod algebra is $H^{*}(k o) \cong$ $\mathcal{A}_{2} \otimes_{\mathcal{A}(1)} \mathbf{Z} / 2$. The Künneth homomorphism

$$
H^{*}(k o \wedge X) \cong\left(\mathcal{A}_{2} \otimes_{\mathcal{A}(1)} \mathbf{Z} / 2\right) \otimes H^{*}(X) \cong \mathcal{A}_{2} \otimes_{\mathcal{A}(1)} H^{*}(X)
$$

and the ring change formula $\operatorname{Hom}_{\mathcal{A}_{2}}\left(\mathcal{A}_{2} \otimes_{\mathcal{A}(1)} M, N\right) \cong \operatorname{Hom}_{\mathcal{A}(1)}(M, N)$ turn the ordinary mod 2 Adams spectral sequence into the one with the $E_{2}$-term

$$
\operatorname{Ext}_{\mathcal{A}(1)}^{s, t}\left(H^{*}(X), \mathbf{Z} / 2\right) \Longrightarrow k o_{t-s}(X) .
$$

Here we use regular conventions to draw the cell-diagrams for the spectra in question. Recall that 


$$
H^{*}(k o)=\mathcal{A}_{2} \otimes_{\mathcal{A}(1)} \text { • and } H^{*}(k o\langle 2\rangle)=\mathcal{A}_{2} \otimes_{\mathcal{A}(1)} \text { (the joker) }
$$

Let $k(1)$ be a connected cover of the first Morava $k$-theory spectrum $K(1)$ with the coefficient ring $k(1)_{*} \cong \mathbf{Z} / 2\left[v_{1}\right]$. Here is the result for the spectra $k o^{\eta}$, $k o^{\Sigma_{2}}$ :

Lemma 5.5 There are the following homotopy equivalences

$$
k o^{\eta} \cong k u, \quad k o^{\Sigma_{2}} \cong k(1)
$$

The following result one can prove by an easy computation:

Lemma 5.6 There are isomorphisms of the following $\mathcal{A}(1)$-modules:

$$
\text { (?) }
$$

Using the Adams spectral sequence for the spectra $k o\langle 2\rangle^{\eta}$ and $k o\langle 2\rangle^{\Sigma_{2}}$, one obtains the following result:

Lemma 5.7 There are the following homotopy equivalences

$$
\begin{aligned}
& k o\langle 2\rangle^{\eta} \cong \mathbf{H}(\mathbf{Z} / 2) \vee \Sigma^{2} k u, \\
& k o\langle 2\rangle^{\Sigma_{2}} \cong \mathbf{H}(\mathbf{Z} / 2) \vee \Sigma \mathbf{H}(\mathbf{Z} / 2) \vee \Sigma^{2} k(1) .
\end{aligned}
$$

It is convenient to denote:

$$
\begin{aligned}
& \widehat{k o}=\prod_{1 \notin I,} \Sigma^{4 n(I)} k o \times \prod_{1 \notin I,} \Sigma^{4 n(I)-4} k o\langle 2\rangle, \quad \text { and } \quad \mathbf{H} \widehat{(\mathbf{Z} / 2)}=\prod_{k} \Sigma^{\operatorname{deg} z_{k}} \mathbf{H}(\mathbf{Z} / 2) . \\
& n(I) \neq 0 \text {, even } \quad n(I) \text { odd }
\end{aligned}
$$

The spectra $\widehat{k o}^{\Sigma}$ are defined similarly for $\Sigma=\Sigma_{1}, \Sigma_{2}, \Sigma_{3}$ or $\eta$. Theorem 5.2 implies the following result: 
Corollary 5.8 There is the following homotopy equivalence of 2-local spectra:

$$
F^{\Sigma}: M \operatorname{Spin}^{\Sigma} \longrightarrow k o^{\Sigma} \vee \widehat{k o}^{\Sigma} \vee \mathbf{H} \widehat{(\mathbf{Z} / 2)^{\Sigma}} \text {, where } \Sigma=\Sigma_{1}, \Sigma_{2} \text {, or } \eta \text {. }
$$

Remark 5.9 The coefficient groups of the $K$-theories $K O^{\Sigma}$ are well-known in homotopy theory. We give the table of the groups $K O_{n}^{\Sigma_{1}}=K O_{n}(p t, \mathbf{Z} / 2)$ for convenience:

\begin{tabular}{|c|c|c|c|c|c|c|c|c|c|c|}
\hline & 0 & 1 & 2 & 3 & 4 & 5 & 6 & 7 & 8 & \\
\hline$K O_{n}^{\Sigma_{1}}=K O_{n}(p t, \mathbf{Z} / 2)$ & $\mathbf{Z} / 2$ & $\mathbf{Z} / 2$ & $\mathbf{Z} / 4$ & $\mathbf{Z} / 2$ & $\mathbf{Z} / 2$ & 0 & 0 & 0 & $\mathbf{Z} / 2$ & $\ldots$ \\
\hline
\end{tabular}

We emphasize that $K_{8 k+2}(p t, \mathbf{Z} / 2) \cong \mathbf{Z} / 4$.

Remark 5.10 We notice that there is a natural transformation

$$
r: \Omega_{*}^{\operatorname{Spin}_{*} \eta}(\cdot) \longrightarrow \Omega_{*}^{\operatorname{Spin}^{c}}(\cdot) .
$$

Indeed, let $M$ be an $\eta$-manifold, ie, $\partial M \cong \beta_{2} M \times P_{2}$, where $P_{2}=S^{1}$ with nontrivial Spin structure. Then $P_{2}$ is a boundary as a Spin ${ }^{c}$-manifold, even more, $P_{2}=\partial D^{2}$. Then the correspondence

$$
\left(M, \partial M=\beta_{2} M \times P_{2}\right) \mapsto\left(N=M \cup-\beta_{2} M \times D^{2}\right)
$$

determines the transformation $r$. In particular, $r$ gives a map of classifying spectra: $r: M$ Spin $^{\eta} \longrightarrow M$ Spin $^{c}$. It is easy to see that there is a commutative diagram

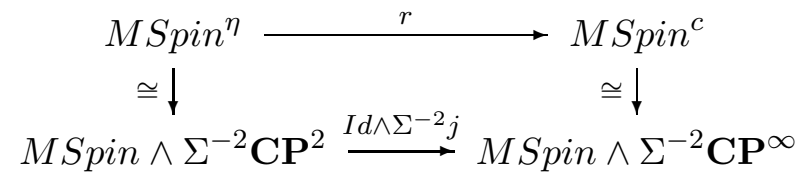

where $j: \mathbf{C P}^{2} \longrightarrow \mathbf{C P}^{\infty}$ is the standard embedding. There are simple geometric reasons which imply that the transformation $r$ is not multiplicative. In fact, it is very similar to the transformation $\Omega_{*}^{S U, \eta}(\cdot) \longrightarrow \Omega_{*}^{U}(\cdot)$, where $\Omega_{*}^{S U, \eta}(\cdot)$ is the $S U$-cobordism theory with $\eta$-singularities. The cobordism theory $\Omega_{*}^{S U, \eta}(\cdot)$ may be easily identified with the Conner-Floyd theory $W(\mathbf{C}, 2)_{*}(\cdot)$, see [19].

\section{$6 \quad$ The spectrum $M \operatorname{Spin}^{\Sigma_{3}}$}

Let $A: \Sigma^{8} M(2) \longrightarrow M(2)$ be the Adams map. Let $V(1)$ be a cofiber:

$$
\Sigma^{8} M(2) \stackrel{A}{\longrightarrow} M(2) \stackrel{p}{\longrightarrow} V(1) .
$$

The objective of this section is to prove the following result. 
Theorem 6.1 There is a homotopy equivalence of spectra localized at 2:

$$
M \operatorname{Spin}^{\Sigma_{3}} \cong M \operatorname{Spin} \wedge \Sigma^{-2} \mathbf{C P}^{2} \wedge V(1) \text {. }
$$

Proof Recall that the Adams map $A$ induces a multiplication by the Bott element in $K O_{*}$ and connected covers $k o_{*}$ and $k o\langle 2\rangle_{*}$. Let, as above, $Y=$ $\Sigma^{-2} \mathbf{C P}^{2} \wedge M(2)$. We apply the Cartan formula (12)



to obtain the formula:

$$
\begin{aligned}
& M \operatorname{Spin} \wedge M \operatorname{Spin}^{\Sigma_{2}} \stackrel{\mu_{\Sigma_{2}}}{\longrightarrow} \operatorname{MSpin}^{\Sigma_{2}} \\
& \sum\left(\pi^{I_{1}} \wedge \pi_{\Sigma_{2}}^{I_{2}}\right) \downarrow \quad \pi_{\Sigma_{2}}^{I} \downarrow \quad \text { or } \mu_{\Sigma_{2}} \pi_{\Sigma_{2}}^{I}=\sum_{I_{1}+I_{2}=I} \mu_{\Sigma_{2}}^{\prime}\left(\pi^{I_{1}} \wedge \pi_{\Sigma_{2}}^{I_{2}}\right) . \\
& K O \wedge K O^{\Sigma_{2}} \stackrel{\mu_{\Sigma_{2}}^{\prime}}{\longrightarrow} K O^{\Sigma_{2}}
\end{aligned}
$$

Let $X$ be a space, $x \in \Omega_{*}^{\text {Spin }}(X), b \in \Omega_{8}^{\text {Spin }}$ be the Bott element. Then $\mu_{\Sigma_{2}}(b, x)=b \cdot x \in \Omega_{*+8}^{\text {Spin }}(X)$.

Lemma 6.2 The $K O^{\Sigma_{2}}$-characteristic numbers $\pi_{\Sigma_{2}}^{I}: M \operatorname{Spin}^{\Sigma_{2}} \longrightarrow K O^{\Sigma_{2}}$ commutes with a multiplication by the Bott element, ie, $\pi_{\Sigma_{2}}^{I}(b \cdot x)=b \cdot \pi_{\Sigma_{2}}^{I}(x)$.

Proof The Cartan formula (18) and Lemma 5.3 gives:

$$
\begin{aligned}
& \pi_{\Sigma_{2}}^{I}(b \cdot x)=\sum_{I_{1}+I_{2}=I}\left(\pi^{I_{1}}(b) \pi_{\Sigma_{2}}^{I_{2}}(x)=b \cdot \pi_{\Sigma_{2}}^{I}(x)+\pi^{(1)}(b) y+\pi^{(1,1)}(b) z=\right. \\
& b \cdot \pi_{\Sigma_{2}}^{I}(x)+(2 c) \cdot y+(2 d) \cdot z=b \cdot \pi_{\Sigma_{2}}^{I}(x)+c \cdot(2 y)+d \cdot(2 z)=b \cdot \pi_{\Sigma_{2}}^{I}(x) .
\end{aligned}
$$

Here $\pi^{(1)}(b)=2 c, y, z \in K O_{*}^{\Sigma_{2}}(X)$, and $\pi^{(1,1)}(b)=2 d$ by Lemma 5.3. We note that $2 y=0$ and $2 z=0$ since the cobordism theory $\Omega_{*}^{S p i n, \Sigma_{2}}(\cdot)$ has an admissible product structure by Theorem 4.1.

Let $I$ be a partition, and $1 \notin I$. The map $\pi_{\Sigma_{2}}^{I}: M \operatorname{Spin}_{\Sigma_{2}} \longrightarrow K O_{\Sigma_{2}}$ lifts to connective cover: $\bar{\pi}_{\Sigma_{2}}^{I}: M \operatorname{Spin}^{\Sigma_{2}} \longrightarrow k o\langle 4 n(I)\rangle^{\Sigma_{2}}$. Let $S^{8} \stackrel{b}{\longrightarrow} M S p i n$ be a map representing the Bott element $b \in \Omega_{8}^{S p i n}$. We denote by $\cdot b$ the composition

$$
\Sigma^{8} M \operatorname{Spin}=S^{8} \wedge M \operatorname{Spin}^{\Sigma_{2}} \stackrel{b \wedge 1}{\longrightarrow} M \operatorname{Spin} \wedge M \operatorname{Spin}^{\Sigma_{2}} \stackrel{\mu^{\prime \prime}}{\longrightarrow} M \operatorname{Spin}^{\Sigma_{2}} .
$$


Note that the diagram

$$
\begin{aligned}
\Sigma^{8} M \operatorname{Spin}^{\Sigma_{2}} & M^{\cdot b} \operatorname{Spin}^{\Sigma_{2}} \\
\cong \downarrow & \cong \downarrow \\
\Sigma^{8} M \operatorname{Spin} \wedge & M(2) \wedge \Sigma^{-2} \mathbf{C P}^{2} \stackrel{\cdot b \wedge 1 \wedge 1}{\longrightarrow} M \operatorname{Spin} \wedge M(2) \wedge \Sigma^{-2} \mathbf{C P}^{2}
\end{aligned}
$$

commutes since $M S \sin ^{\Sigma_{2}}$ is a module over MSpin.

Lemma 6.3 Let $I$ be a partition, so that $1 \notin I$. The following diagrams commute:

$$
\begin{aligned}
& \Sigma^{8} M \operatorname{Spin}^{\Sigma_{2}} \longrightarrow \operatorname{MSpin}^{\Sigma_{2}} \\
& \begin{array}{rr}
\downarrow^{8} \pi_{\Sigma_{2}}^{I} & k o\langle n(I)\rangle^{\Sigma_{2}} \\
\Sigma^{8} k o\langle n(I)\rangle^{\Sigma_{2}} & \downarrow \pi_{\Sigma_{2}}^{I}
\end{array} \\
& \downarrow \cong \\
& \downarrow \cong \\
& k o\langle n(I)\rangle \wedge \Sigma^{8} M(2) \wedge \Sigma^{-2} \mathbf{C P}^{2} \stackrel{1 \wedge A \wedge 1}{\longrightarrow} k o\langle n(I)\rangle \wedge M(2) \wedge \Sigma^{-2} \mathbf{C P}^{2} \\
& \begin{array}{rl}
\Sigma^{8} M \operatorname{Spin}^{\Sigma_{2}} & \cdot b \\
\downarrow \Sigma^{8} z_{k}^{\Sigma_{2}} & M \operatorname{Spin}^{\Sigma_{2}} \\
\downarrow \Sigma^{8} z_{k}^{\Sigma_{2}}
\end{array} \\
& \Sigma^{8+\operatorname{deg} z_{k}} \mathbf{H}(\mathbf{Z} / 2) \wedge M(2) \wedge \Sigma^{-2} \mathbf{C P}^{2} \quad \Sigma^{\operatorname{deg} z_{k}} \mathbf{H}(\mathbf{Z} / 2) \wedge M(2) \wedge \Sigma^{-2} \mathbf{C P}^{2} \\
& \downarrow \cong \downarrow \cong \\
& \Sigma^{\operatorname{deg} z_{k}} \mathbf{H}(\mathbf{Z} / 2) \wedge \Sigma^{8} M(2) \wedge \Sigma^{-2} \mathbf{C P}^{2} \stackrel{1 \wedge A \wedge 1}{\longrightarrow} \Sigma^{\operatorname{deg} z_{k}} \mathbf{H}(\mathbf{Z} / 2) \wedge M(2) \wedge \Sigma^{-2} \mathbf{C P}^{2}
\end{aligned}
$$

Proof A commutativity of the first diagram follows from Lemma 6.2 and the diagram (19). Recall that a projection of the Bott element into the homotopy group of $\Sigma^{\operatorname{deg} z_{k}} \mathbf{H}(\mathbf{Z} / 2)$ is zero. Let $X$ be a finite spectrum. The map

$$
\begin{aligned}
1 \wedge A \wedge 1 \wedge 1: \Sigma^{\operatorname{deg} z_{k}} \mathbf{H}(\mathbf{Z} / 2) & \wedge \Sigma^{8} M(2) \wedge \Sigma^{-2} \mathbf{C P}^{2} \wedge X \longrightarrow \\
& \Sigma^{\operatorname{deg} z_{k}} \mathbf{H}(\mathbf{Z} / 2) \wedge M(2) \wedge \Sigma^{-2} \mathbf{C P}^{2} \wedge X
\end{aligned}
$$

in homotopy coincides with the homomorphism in mod 2 homology groups

$$
\Sigma^{\operatorname{deg} z_{k}} H_{*}\left(\Sigma^{8} M(2) \wedge \Sigma^{-2} \mathbf{C P}^{2} \wedge X\right) \stackrel{A_{*} \otimes 1 \otimes 1}{\longrightarrow} \Sigma^{\operatorname{deg} z_{k}} H_{*}\left(M(2) \wedge \Sigma^{-2} \mathbf{C P}^{2} \wedge X\right)
$$

and is trivial for any space $X$ since $A$ has the Adams filtration 4. It implies that $1 \wedge A \wedge 1$ is a trivial map. A commutativity of (20) now follows.

To complete the proof of Theorem 6.1 we notice that Lemmas 6.3 and 6.2 give the commutative diagram

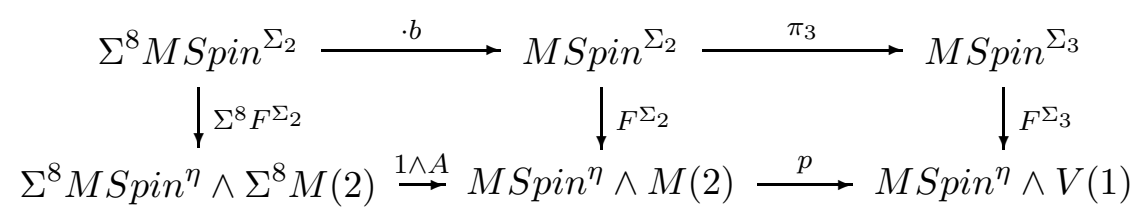


where the map $F^{\Sigma_{3}}$ exists since the both rows are cofibrations. The five-lemma implies that $F^{\Sigma_{3}}$ is a homotopy equivalence.

Corollary 6.4 The spectrum $K O^{\Sigma_{3}}=K O \wedge \Sigma^{-2} \mathbf{C P}^{2} \wedge V(1)$ is a contractible spectrum.

Remark 6.5 The connective spectrum $k o^{\Sigma_{3}}$ is of some interest. It is certainly not contractible, and it is very easy to see that

$$
k o_{j}^{\Sigma_{3}}=\left\{\begin{array}{cl}
\mathbf{Z} / 2 & \text { if } j=0,2,4,6 \\
0 & \text { otherwise }
\end{array}\right.
$$

and the Postnikov tower of $k o^{\Sigma_{3}}$ has the operation $Q_{1}$ as its $k$-invariants.

The technique we used above may be applied to prove the following result:

Corollary 6.6 There is such admissible product structure $\mu^{(2)}$ of the spectrum MSpin ${ }^{\Sigma_{2}}$, so that the map $\bar{\pi}_{\Sigma_{2}}^{0}: M \operatorname{Spin}^{\Sigma_{2}} \longrightarrow k o^{\Sigma_{2}}=k(1)$ is a ring spectra map, moreover, there is an inverse ring spectra map $\rho_{\Sigma_{2}}^{0}: k o^{\Sigma_{2}} \longrightarrow$ $M \operatorname{Spin}^{\Sigma_{2}}$. In other words, $k^{\Sigma_{2}}$ splits off of the spectrum MSpin ${ }^{\Sigma_{2}}$ as a ring spectrum.

\section{Surgery Lemma for $\Sigma-$ manifolds}

7.1 A Riemannian metric on a $\Sigma$-manifold Here we describe what do we mean by a Riemannian metric on manifold with singularities. We consider the case when a manifold has of at most three singularities, $\Sigma_{3}=\left(P_{1}, P_{2}, P_{3}\right)$. We denote $\Sigma_{1}=\left(P_{1}\right), \Sigma_{2}=\left(P_{1}, P_{2}\right)$. We assume that there are given Riemannian metrics $g_{P_{i}}$ on the manifolds $P_{i}, i=1,2,3$. As we mentioned earlier, the metrics $g_{P_{i}}$ are not assumed to be psc-metrics.

If $M$ is a $\Sigma_{3}$-manifold, we assume that it is given a decomposition of the boundary $\partial M$ :

$$
\begin{aligned}
& \partial M=\left(\beta_{1} M \times P_{1} \cup \beta_{2} M \times P_{2} \cup \beta_{2} M \times P_{2}\right) \cup \beta_{123} M \times P_{1} \times P_{3} \times P_{2} \times D^{2} \\
& \cup\left(\beta_{12} M \times P_{1} \times P_{2} \times I_{12} \cup \beta_{23} M \times P_{2} \times P_{3} \times I_{23} \cup \beta_{13} M \times P_{1} \times P_{3} \times I_{13}\right)
\end{aligned}
$$

glued together as it is shown on Figure 7 (a). We start with a Riemannian metric $g_{123}$ on the manifold $\beta_{123} M$. We assume that the manifold

$$
\beta_{123} M \times P_{1} \times P_{2} \times P_{3} \times D^{2}
$$


has product metric $g_{123} \times g_{P_{1}} \times g_{P_{2}} \times g_{P_{3}} \times g_{0}$, where $g_{0}$ is the standard flat metric on the disk $D^{2}$.

Besides, we assume that the manifold $\beta_{123} M \times P_{1} \times P_{2} \times P_{3}$, being common boundary of the manifolds

$$
\beta_{12} M \times P_{1} \times P_{2}, \quad \beta_{13} M \times P_{1} \times P_{3}, \quad \text { and } \quad \beta_{23} M \times P_{2} \times P_{3},
$$

is embedded together with the colors (see Figure $7(\mathrm{a})$ ):

$$
\begin{aligned}
& \beta_{123} M \times P_{1} \times P_{2} \times P_{3} \times I_{12}^{\prime} \subset \beta_{12} M \times P_{1} \times P_{2}, \\
& \beta_{123} M \times P_{1} \times P_{2} \times P_{3} \times I_{13}^{\prime} \subset \beta_{13} M \times P_{1} \times P_{3}, \\
& \beta_{123} M \times P_{1} \times P_{2} \times P_{3} \times I_{23}^{\prime} \subset \beta_{23} M \times P_{2} \times P_{3} .
\end{aligned}
$$

Here $I_{i j}^{\prime}$ are the intervals embedded into the flat disk $D^{2}$ as it is shown on Figure 7 (b).

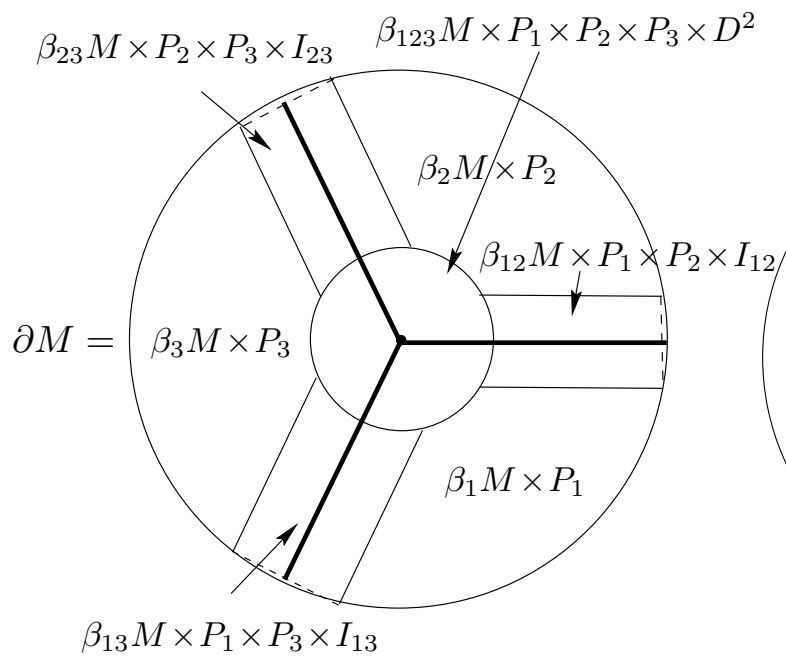

(a) The decomposition of $\partial M$

Figure 7

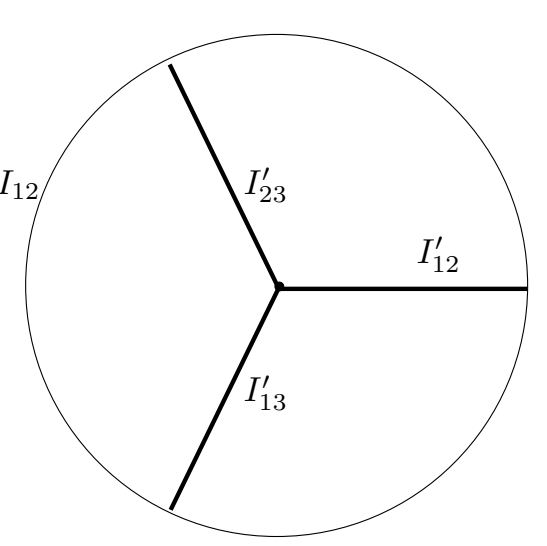

(b) Flat disk $D^{2}$

Let $g_{i j}$ be metrics on the manifolds $\beta_{i j} M$. We assume that the product metric

$$
g_{i j} \times g_{P_{i}} \times g_{P_{j}} \text { on the manifold } \beta_{i j} M \times P_{i} \times P_{j}
$$

coincides with the product metric on the color $\beta_{123} M \times P_{1} \times P_{2} \times P_{3} \times I_{i j}^{\prime}$ near its boundary. Finally if $g_{i}$ is a metric in $\beta_{i} M(i=1,2,3)$, then we assume that the product metric $g_{i} \times g_{P_{i}}$ on $\beta_{i} M \times P_{i}$ coincides with the above product metrics on the manifold $\beta_{i j} M \times g_{P_{i}} \times g_{P_{j}} \times I_{i j}$. Furthermore, the product metric $g_{i} \times g_{P_{i}}$ on $\beta_{i} M \times P_{i}$ restricted on the manifold

$$
\left(\beta_{123} M \times P_{1} \times P_{2} \times P_{3} \times D^{2}\right) \cap\left(\beta_{i} M \times P_{i}\right)
$$


coincides with the product metric $g_{123} \times g_{P_{1}} \times g_{P_{2}} \times g_{P_{3}} \times g_{0}$. Finally the metric $g$ on the manifold $M$ is assumed to be product metric near the boundary $\partial M$. Let $M$, as above be a $\Sigma$-manifold with the same singularities $\Sigma=\left(P_{1}, P_{2}, P_{3}\right)$. We say that a metric $g$ on $M$ is of positive scalar curvature, if, besides the above conditions, the metrics $g$ on $M, g_{i}$ on $\beta_{i} M, g_{i j}$ on $\beta_{i j} M$, and $g_{123}$ on $\beta_{123} M$ have positive scalar curvature functions.

7.2 Surgery theorem in the case of manifolds without singularities Here we briefly review key results on the connection between positive scalar curvature metric and surgery for manifolds without singularities. The first basic result is due to Gromov-Lawson [9, Theorem A] and to Schoen-Yau [27]. A detailed "textbook" proof may be found in [25, Theorem 3.1].

Theorem 7.1 (Gromov-Lawson [9], Schoen-Yau [27]) Let $M$ be a closed manifold, not necessarily connected, with a Riemannian metric of positive scalar curvature, and let $M^{\prime}$ is obtained from $M$ by a surgery of codimension $\geq 3$. Then $M^{\prime}$ also admits a metric of positive scalar curvature.

To get started with $\Sigma$-manifolds we need an "improved version" of Theorem 7.1 which is due to Gajer [8].

Theorem 7.2 (Gajer [8]) Let $M$ be a closed manifold, not necessarily connected, with a Riemannian metric $g$ of positive scalar curvature, and let $M^{\prime}$ is obtained from $M$ by a surgery of codimension $\geq 3$. Then $M^{\prime}$ also admits a metric $g^{\prime}$ of positive scalar curvature. Furthermore, let $W$ be the trace of this surgery (ie, a cobordism $W$ with $\partial W=M \sqcup-M^{\prime}$ ). Then there is a positive scalar curvature metric $\bar{g}$ on $W$, so that $\bar{g}=g+d t^{2}$ near $M$ and $\bar{g}=g^{\prime}+d t^{2}$ near $M^{\prime}$.

In order to use the above Surgery Theorems, one has to specify certain structure of manifolds under consideration. This structure (known as $\gamma$-structure) is determined by the fundamental group $\pi_{1}(M)$, and the Stiefel-Whitney classes $w_{1}(M)$, and $w_{2}(M)$. Indeed, it is well-known that the fundamental group $\pi$ is crucially important for the existence question. Then there is clear difference when a manifold $M$ is oriented or not (which depends on $w_{1}(M)$ ). On the other hand, a presence of the Spin-structure (which means that $w_{2}(M)=0$ ) gives a way to use the Dirac operator on $M$ to control the scalar curvature via the vanishing formulas. Stolz puts together those invariants to define a $\gamma$-structure, see [31]. In the case we are interested in, all manifolds are simplyconnected and Spin, thus we will state only a relevant Bordism Theorem (see, say, [25, Theorem 4.2] for a general result). 
Theorem 7.3 Let $M$ be a simply connected Spin manifold, $\operatorname{dim} M \geq 5$. Then $M$ admits a metric of positive scalar curvature if and only if there is some simply-connected Spin-manifold $M^{\prime}$ of positive scalar curvature in the same Spin-bordism class.

7.3 Surgery theorem in the case of manifolds with singularities Let $M$ be a $\Sigma$-manifold with $\Sigma=\left(P_{i}\right),\left(P_{i}, P_{j}\right)$ or $\left(P_{i}, P_{j}, P_{k}\right)$. Here $P_{i}$ are arbitrary closed manifolds. Let $\operatorname{dim} M=n$, and $\operatorname{dim} P_{i}=p_{i}, i=1,2,3$. Then we denote $\operatorname{dim} \beta_{i} M=n_{i}=n-p_{i}-1, \operatorname{dim} \beta_{i j} M=n_{i j}=n-p_{i}-p_{j}-2$, and $\operatorname{dim} \beta_{123} M=n_{123}=n-p_{1}-p_{2}-p_{3}-3$. The manifolds $\beta_{i} M, \beta_{i j} M$ and $\beta_{i j k} M$ are called $\Sigma$-strata of $M$.

We say that a $\Sigma$-manifold $M$ is simply connected if $M$ itself is simply connected and all $\Sigma$-strata of $M$ are simply connected manifolds.

Theorem 7.4 Let $M$ be a simply connected Spin $\Sigma$-manifold, $\operatorname{dim} M=$ $n$, so that all $\Sigma$-strata manifolds are nonempty, and satisfying the following conditions:

(1) if $\Sigma=\left(P_{i}\right)$, then $n-p_{i} \geq 6$;

(2) if $\Sigma=\left(P_{i}, P_{j}\right)$, then $n-p_{i}-p_{j} \geq 7$;

(3) if $\Sigma=\left(P_{i}, P_{j}, P_{k}\right)$, then $n-p_{i}-p_{j}-p_{k} \geq 8$.

Then $M$ admits a positive scalar curvature if and only if there is some simplyconnected Spin ${ }^{\Sigma}$-manifold $M^{\prime}$ of positive scalar curvature in the same $\operatorname{Spin}^{\Sigma_{-}}$ bordism class.

Remark 7.5 The role of the manifolds $M$ and $M^{\prime}$ are not symmetric here. For instance, it is important that $M$ has all $\Sigma$-strata manifolds nonempty, however, the manifold $M^{\prime}$ may have empty singularities.

Proof (1) Let $W$ be a $\operatorname{Spin}^{\Sigma}$-cobordism between $M$ and $M^{\prime}$. Then $\beta_{i} W$ is a Spin-cobordism between $\beta_{i} M$ and $\beta_{i} M^{\prime}$. By condition, $\beta_{i} M^{\prime}$ is simply connected, and $\operatorname{dim} \beta_{i} M^{\prime}=\operatorname{dim} M \geq 5$. We notice that there is a sequence of surgeries on the manifold $\beta_{i} W$ (relative to the boundary $\partial \beta_{i} M^{\prime}$ ) so that the resulting manifold is $2-$ connected (see an argument given in $[9$, Proof of Theorem A]). Let $V$ be a trace of this surgery. Then its boundary is decomposed as

$$
\partial V=\beta_{i} W \cup\left(\beta_{i} M \times I\right) \cup\left(\beta_{i} M^{\prime} \times I\right) \cup L_{i}
$$


We glue together the manifolds $W$ and $-V \times P_{i}$ :

$$
W^{\prime}:=W \cup_{\beta_{i} W \times P_{i}}-V \times P_{i} .
$$

Then the boundary of $W W^{\prime}$ (as a $\operatorname{Spin}^{\Sigma}$-manifold) is

$$
\begin{aligned}
& \delta W^{\prime}=\left(M \cup\left(\beta_{i} M \times I \times P_{i}\right)\right) \sqcup\left(M^{\prime} \cup\left(\beta_{i} M^{\prime} \times I \times P_{i}\right)\right) \cong M \sqcup M^{\prime}, \\
& \text { and } \beta_{i} W^{\prime}=L_{i}, \quad \text { with } \partial L_{i}=\beta_{i} M \sqcup \beta_{i} M^{\prime} .
\end{aligned}
$$

Now we use Theorem 7.2 to "push" a positive scalar curvature metric from $\beta_{i} M^{\prime}$ through $L_{i}$ to $\beta_{i} M$ keeping it a product metric near the boundary. At this point a psc-metric $g_{i}$ on $L_{i}$ may be such that the product metric $g_{i} \times g_{P_{i}}$ is not of positive scalar curvature. We find $\epsilon>0$ so that the product metric $\epsilon g_{i} \times g_{P_{i}}$ has positive scalar curvature, and then we attach one more cylinder $L_{i} \times P_{i} \times[0, a]$ with the metric

$$
g_{i}(t):=\frac{a-t}{a} g_{i} \times g_{P_{i}}+\frac{t}{a} \epsilon g_{i} \times g_{P_{i}}+d t^{2} .
$$

We use metric $g_{i}(t)$ to fit together the metric already constructed on $W^{\prime \prime}$ with the metric on $L_{i} \times P_{i} \times[0, a]$. In particular, there is $a>0$ so that the restriction of $g_{i}(t)$ on $\beta_{i} M^{\prime} \times P_{i} \times[0, a]$ has positive scalar curvature (since an isotopy of positive scalar curvature metrics implies concordance). By small perturbation, we can change $g_{i}(t)$, so that it has positive scalar curvature and it is a product near the boundary. Then we do surgeries on the interior of $W^{\prime}$ to make it 2connected. Let $W^{\prime \prime}$ be the resulting manifold. In particular, $\beta_{i} W^{\prime \prime}=\beta_{i} W^{\prime}=$ $L_{i}$. Finally we use "push" a positive scalar curvature metric from $M^{\prime}$ to $M$ through $W^{\prime \prime}$ keeping it a product metric near the singular stratum $\beta_{i} \beta_{i} W^{\prime}=L_{i}$.

(2) Let $M$ be a simply connected Spin $\Sigma$-manifold, with $\Sigma=\left(P_{i}, P_{j}\right)$, and $n-p_{i}-p_{j} \geq 7$. By condition, the singular stratum $\beta_{i j} M \neq \emptyset$. Let $W$ be

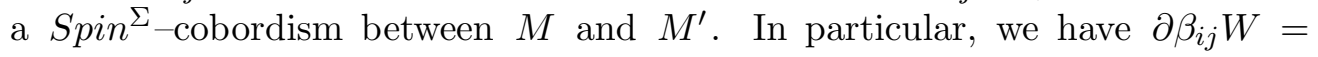
$\beta_{i j} M \sqcup \beta_{i j} M^{\prime}$. Recall that $\beta_{i j} W \times P_{i} \times P_{j}$ is embedded to the union

$$
\left(\beta_{i} W \times P_{i}\right) \cup\left(\beta_{j} W \times P_{j}\right)
$$

together with the colors

$$
\beta_{i j} W \times P_{i} \times P_{j} \times[-\epsilon, \epsilon]
$$

By conditions, the manifolds $\beta_{i j} M \beta_{i j} M^{\prime}$ are simply connected, and $\operatorname{dim} \beta_{i j} M$ $=\operatorname{dim} \beta_{i j} M^{\prime} \geq 5$. As above, there is a surgery on $\beta_{i j} W$ (relative to the boundary $\partial \beta_{i j} W=\beta_{i j} M \sqcup \beta_{i j} M^{\prime}$ ) so that a resulting manifold is 2-connected. Let $V_{i j}$ be the trace of this surgery:

$$
\partial V=\beta_{i j} W \cup\left(\beta_{i j} M \times I\right) \cup\left(\beta_{i j} M^{\prime} \times I\right) \cup L_{i j} .
$$


We glue together the manifolds

$$
W \quad \text { and } \quad-V \times[-\epsilon, \epsilon] \times P_{i} \times P_{j}
$$

to obtain a manifold $W^{\prime}$, where we identify

$$
\begin{aligned}
& \beta_{i j} W \times P_{i} \times P_{j} \times[-\epsilon, \epsilon] \subset\left(\beta_{i} W \times P_{i}\right) \cup\left(\beta_{j} W \times P_{j}\right) \text { and } \\
& -\beta_{i j} W \times P_{i} \times P_{j} \times[-\epsilon, \epsilon] \subset-\partial V \times[-\epsilon, \epsilon] \times P_{i} \times P_{j},
\end{aligned}
$$

see Figure 8.

The resulting manifold $W^{\prime}$ (after smoothing corners and extending metric according with the Surgery Theorem construction) is such that $\beta_{i j} W^{\prime}=L_{i j}$ is 2 -connected cobordism between $\beta_{i j} M$ and $\beta_{i j} M^{\prime}$. Thus we can "push" a positive scalar curvature metric from $\beta_{i j} M^{\prime}$ to $\beta_{i j} M$ through the cobordism $\beta_{i j} W^{\prime}$. Thus we obtain a psc-metric $g_{i j}$ on $\beta_{i j} M^{\prime}$ which is a product near boundary. In general, the product metric $g_{i j} \times g_{P_{i}} \times g_{P_{j}}$ on $\beta_{i j} W \times P_{i} \times P_{j}$ is not of positive scalar curvature. Then we have to attach one more cylinder

$$
\beta_{i j} W^{\prime} \times[-\epsilon, \epsilon] \times I \times P_{i} \times P_{j}
$$

to "scale" the metric $g_{i j} \times g_{P_{i}} \times g_{P_{j}}$ to a positive scalar curvature metric $\epsilon_{i j} g_{i j} \times$ $g_{P_{i}} \times g_{P_{j}}$ through an appropriate homotopy.
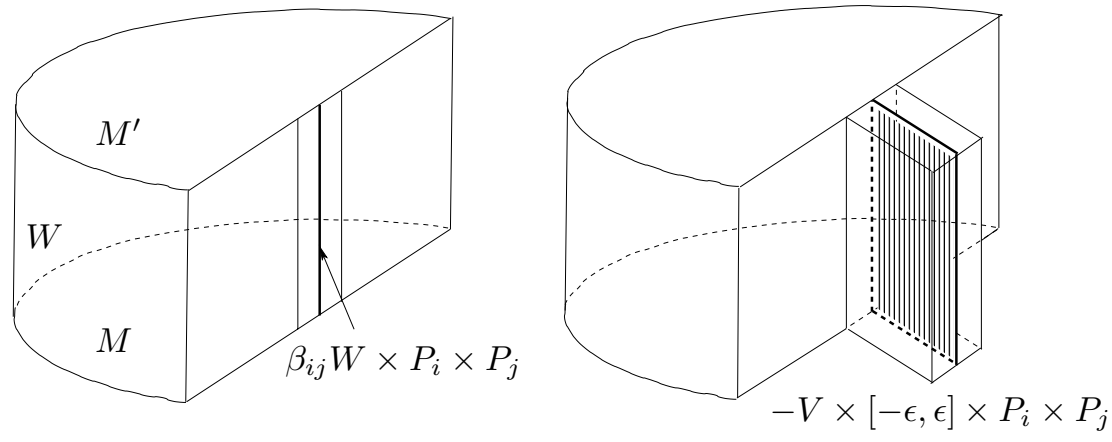

Figure 8

Then we consider the manifolds $\beta_{i} W^{\prime}$ and $\beta_{j} W^{\prime}$. Again, we perform surgeries on the interior of $\beta_{i} W^{\prime}, \beta_{j} W^{\prime}$ to get 2 -connected manifolds $L_{i}$ and $L_{j}$. Let $V_{i}, V_{j}$ be the traces of these surgeries:

$$
\partial V_{i}=\beta_{i} W^{\prime} \cup \beta_{i j} W^{\prime} \times P_{j} \cup L_{i}, \quad \partial V_{j}=\beta_{i} W^{\prime} \cup \beta_{i j} W^{\prime} \times P_{i} \cup L_{j} .
$$

Now we attach the manifolds $-V_{i} \times P_{i}$ and $-V_{j} \times V_{j}$ to $W^{\prime}$ by identifying

$$
\begin{array}{lll}
\beta_{i} W^{\prime} \times P_{i} \subset W^{\prime} & \text { and } & \beta_{j} W^{\prime} \times P_{j} \subset W^{\prime} \quad \text { with } \\
-\beta_{i} W^{\prime} \times P_{i} \subset-\partial V_{i}, & \text { and } & -\beta_{j} W^{\prime} \times P_{j} \subset-\partial V_{j}
\end{array}
$$


respectively. Let $W^{\prime \prime}$ be the resulting manifold (after an appropriate smoothing and extending a metric), see Figure 9. Notice that $W^{\prime \prime}$ is still a $\operatorname{Spin}^{\Sigma_{-}}$ cobordism between $M$ and $M^{\prime}$.

This procedure combined with an appropriate metric homotopy gives $W^{\prime \prime}$ together with a metric $g^{\prime \prime}$ on $W^{\prime \prime}$, so that it is a product metric near the boundary, its restriction on $M^{\prime}$ has positive scalar curvature, and its restriction on the manifolds

$$
\beta_{i} W^{\prime \prime} \times P_{i}, \quad \beta_{j} W^{\prime \prime} \times P_{j}, \quad \beta_{i} W^{\prime \prime} \times[-\epsilon, \epsilon] \times P_{i} \times P_{j}
$$

are psc-metrics

$$
g_{i} \times g_{P_{i}}, \quad g_{j} \times g_{P_{j}} \quad g_{i j} \times g_{P_{i}} \times g_{P_{j}}+d t^{2}
$$

respectively (for some psc-metrics $g_{i}, g_{j}, g_{i j}$ ). It remains to perform surgeries on the interior of $W^{\prime \prime}$ to get a 2-connected manifold, and finally push a pscmetric from $M^{\prime}$ to $M$ relative to the boundary.


Figure 9

A proof of (3) is similar.

\section{Proof of Theorem 1.1}

First we recall the main construction from [29]. Let $G=P S p(3)=S p(3) /(\mathbf{Z} / 2)$, where $\mathbf{Z} / 2$ is the center of $S p(3)$. Let $g_{0}$ be the standard metric on $\mathbf{H P}^{2}$. Recall that the group $G$ acts on $\mathbf{H P}^{2}$ by isometries of the metric $g_{0}$. Let $E \longrightarrow B$ be a geometric $\mathbf{H} \mathbf{P}^{2}$-bundle, ie, $E \longrightarrow B$ is a bundle with a fiber $\mathbf{H P}^{2}$ and structure group $G$. Each geometric $\mathbf{H P}^{2}$-bundle $E \longrightarrow B$ is given by a map 
$f: B \longrightarrow B G$ by taking first the associated principal $G$-bundle, and then by "inserting" $\mathbf{H P}^{2}$ as a fiber employing the action of $G$. Assume that $B$ is a Spin manifold. Then the correspondence $(B, f) \mapsto E$ gives the homomorphism $T: \Omega_{n-8}^{\text {Spin }}(B G) \longrightarrow \Omega_{n}^{\text {Spin }}$. Let $X$ be a finite $C W$-complex. The homomorphism $T$ actually gives the transformation

$$
T: \Omega_{n-8}^{\text {Spin }}\left(X \wedge B G_{+}\right) \longrightarrow \Omega_{n}^{\text {Spin }}(X),
$$

which may be interpreted as the transfer, and induces the map at the level of the classifying spectra

$$
T: M \operatorname{Spin} \wedge \Sigma^{8} B G_{+} \longrightarrow M \operatorname{Spin},
$$

see details in [29]. Consider the composition

$$
\Omega_{n-8}^{\text {Spin }}\left(X \wedge B G_{+}\right) \stackrel{T}{\longrightarrow} \Omega_{n}^{S p i n}(X) \stackrel{\alpha}{\longrightarrow} k o_{n}(X)
$$

Here is the result due to S Stolz, [30]:

Theorem 8.1 Let $X$ be a $C W$-complex. Then there is an isomorphism at the 2-local category:

$$
k o_{n}(X) \cong \Omega_{n}^{S p i n}(X) / \operatorname{Im} T
$$

Let $\Sigma=\Sigma_{1}$ or $\Sigma_{2}$, or $\Sigma_{3}$ or $\eta$, and $X_{\Sigma}$ be the corresponding spectrum, so that $M \operatorname{Spin}^{\Sigma} \cong M \operatorname{Spin} \wedge X_{\Sigma}$. The map T:MSpin $\wedge \Sigma^{8} B G_{+} \longrightarrow M S p i n$ induces the map

$$
T^{\Sigma}: M \operatorname{Spin} \wedge \Sigma^{8} B G_{+} \wedge X_{\Sigma} \longrightarrow M \operatorname{Spin} \wedge X_{\Sigma} .
$$

Consider the composition

$$
M \operatorname{Spin} \wedge \Sigma^{8} B G_{+} \wedge X_{\Sigma} \stackrel{T^{\Sigma}}{\longrightarrow} M \operatorname{Spin} \wedge X_{\Sigma} \stackrel{\alpha^{\Sigma}}{\longrightarrow} k o \wedge X_{\Sigma} .
$$

We use Theorems 3.1 and 6.1 to derive the following conclusion from Theorem 8.1.

Corollary 8.2 Let $\Sigma=\Sigma_{1}$ or $\Sigma_{2}$, or $\Sigma_{3}$ or $\eta$. Then there is an isomorphism at the 2-local category:

$$
k o_{n}^{\Sigma} \cong M \operatorname{Spin}_{n}^{\Sigma} / \operatorname{Im} T^{\Sigma}
$$

We remind here that the homomorphism $k o_{n}^{\Sigma} \rightarrow K O_{n}^{\Sigma}$ is a monomorphism for $n \geq 0$.

Corollary 8.2 describes the situation in 2-local category. Now we consider what is happening when we invert 2. Consider first the case when $\Sigma=\Sigma_{1}$. Then we have a cofibration:

$$
\operatorname{MSpin}\left[\frac{1}{2}\right] \stackrel{\cdot 2}{\longrightarrow} \operatorname{MSpin}\left[\frac{1}{2}\right] \longrightarrow \operatorname{MSpin}^{\Sigma_{1}\left[\frac{1}{2}\right]}
$$


Clearly the map $\cdot 2: \quad M \operatorname{Spin}\left[\frac{1}{2}\right] \rightarrow M \operatorname{Spin}\left[\frac{1}{2}\right]$ is a homotopy equivalence. Thus $\operatorname{MSpin}^{\Sigma_{1}}\left[\frac{1}{2}\right] \cong p t$. The case $\Sigma=(\eta)$ is more interesting. Here we have the cofibration:

$$
S^{1} \wedge \operatorname{MSpin}\left[\frac{1}{2}\right] \stackrel{\cdot \eta}{\longrightarrow} \operatorname{MSpin}\left[\frac{1}{2}\right] \stackrel{\pi^{\eta}}{\longrightarrow} \operatorname{MSpin}^{(\eta)}\left[\frac{1}{2}\right] .
$$

Notice that $\eta=0$ in $p$-local homotopy $\Omega_{1}^{\text {Spin }} \otimes \mathbf{Z}\left[\frac{1}{2}\right]$. Thus we have a short exact sequence:

$$
0 \rightarrow \Omega_{n}^{\text {Spin }} \otimes \mathbf{Z}\left[\frac{1}{2}\right] \stackrel{\pi_{*}^{\eta}}{\longrightarrow} \Omega_{n}^{\text {Spin }, \eta} \otimes \mathbf{Z}\left[\frac{1}{2}\right] \stackrel{\beta}{\longrightarrow} \Omega_{n-2}^{\text {Spin }} \otimes \mathbf{Z}\left[\frac{1}{2}\right] \rightarrow 0 .
$$

This sequence has very simple geometric interpretation. Let $w \in \Omega_{2}^{S p i n, \eta} \cong \mathbf{Z}$ be an element represented by an $(\eta)$-manifold $W$, so that $\beta W=2$. Let $t=\frac{w}{2} \in \Omega_{2}^{S p i n, \eta} \otimes \mathbf{Z}\left[\frac{1}{2}\right]$.

Now let $c \in \Omega_{n}^{\text {Spin, } \eta} \otimes \mathbf{Z}\left[\frac{1}{2}\right]$, and $\beta c=b$. Let $a=c-t b$, then $c=a+t b$ for $a \in \Omega_{n}^{\text {Spin }} \otimes \mathbf{Z}\left[\frac{1}{2}\right], b \in \Omega_{n-2}^{\text {Spin }} \otimes \mathbf{Z}\left[\frac{1}{2}\right]$ for any element $c \in \Omega_{n}^{\text {Spin, },} \otimes \mathbf{Z}\left[\frac{1}{2}\right]$. Furthermore, this decomposition is unique once we choose an element $t$. Recall that $\Omega_{*}^{S p i n} \otimes \mathbf{Z}\left[\frac{1}{2}\right] \cong \Omega_{*}^{S O} \otimes \mathbf{Z}\left[\frac{1}{2}\right]$ is a polynomial algebra $\mathbf{Z}\left[\frac{1}{2}\right]\left[x_{1}, x_{2}, \ldots, x_{j}, \ldots\right]$ with $\operatorname{deg} x_{j}=4 j$. Thus we obtain

$$
\Omega_{n}^{\text {Spin },(\eta)} \otimes \mathbf{Z}\left[\frac{1}{2}\right] \cong \begin{cases}\Omega_{n}^{\text {Spin }} \otimes \mathbf{Z}\left[\frac{1}{2}\right] & \text { if } n=4 k \\ \Omega_{n-2}^{\text {Spin }} \otimes \mathbf{Z}\left[\frac{1}{2}\right] & \text { if } n=4 k+2 \\ 0 & \text { otherwise }\end{cases}
$$

Furthermore, as it is shown in [15, Proposition 4.2] there are generators $x_{j}=$ $\left[M^{4 j}\right]$ of the polynomial algebra $\Omega_{*}^{\text {Spin }} \otimes \mathbf{Z}\left[\frac{1}{2}\right]$, so that the manifolds $M^{4 j}$ are total spaces of geometric $\mathbf{H P}^{2}$-bundles (for all $j \geq 2$ ). In particular, it means that the groups $\Omega_{4 j}^{\text {Spin }} \otimes \mathbf{Z}\left[\frac{1}{2}\right]$ are in the ideal $\operatorname{Im} T \subset \Omega_{*}^{\text {Spin }} \otimes \mathbf{Z}\left[\frac{1}{2}\right]$. Now the formula (23) shows that the groups $\Omega_{n}^{\text {Spin, }(\eta)} \otimes \mathbf{Z}\left[\frac{1}{2}\right]$ are in the ideal $\operatorname{Im} T^{\eta}$. We obtain the isomorphism in integral homotopy groups: $k o_{n}^{\Sigma} \cong \Omega_{n}^{S p i n, \eta} / \operatorname{Im} T^{\eta}$.

The cases $\Sigma=\Sigma_{2}$ or $\Sigma=\Sigma_{3}$ are similar to the case $\Sigma=\Sigma_{1}$ : here we have that $\operatorname{MSpin}^{\Sigma_{i}}\left[\frac{1}{2}\right] \cong p$ for $i=2,3$.

Thus in all cases we conclude that any element $x \in \operatorname{Im} T^{\Sigma}$ may be represented by a simply connected $\Sigma$-manifold admitting a psc-metric. Here the restriction that $\operatorname{dim} x \geq d(\Sigma)$ is essential. Thus we conclude that if a simply-connected Spin $\Sigma$ manifold $M$ with $\operatorname{dim} M \geq d(\Sigma)$ is such that $[M] \in \operatorname{Ker} \alpha^{\Sigma}$, then $M$ admits a psc-metric.

Now we prove the necessity. 
Let $M$ be a simply connected Spin-manifold of dimension $\operatorname{dim} M \geq d(\Sigma)$. What we really must show is that if there is a psc-metric on $M$, then $\alpha^{\Sigma}([M])=$ 0 on the group $K O_{*}^{\Sigma}$.

The case $\Sigma_{1}=\left(P_{1}\right)=\langle 2\rangle$ is done in [22], where it is shown that $\alpha^{\Sigma}([M]) \in$ $K O_{*}^{\langle 2\rangle}$ coincides with the index of the Dirac operator on $M$, and that the index $\alpha^{\Sigma}([M])$ vanishes if $M$ has a psc-metric.

The next case to consider is when $\Sigma=\eta=\left(P_{2}\right)$. Let $M$ be a closed $\Sigma$ manifold, ie, $\partial M=\beta_{2} M \times P_{2}$, where $P_{2}$ is a circle with the nontrivial Spin structure. Let $g$ be a psc-metric on $M$. In particular, we have a psc-metric $g_{\beta_{2} M}$. Then, as we noticed earlier, the circle $P_{2}$ is zero-cobordant as $\operatorname{Spin}^{c}-$ manifold. More precisely, we choose a disk $D^{2}$ with $\partial D^{2}=P_{2}$, and construct the manifold

$$
\bar{M}=M \cup\left(-\beta_{2} M \times D^{2}\right)
$$

where we identify $\partial M \beta_{2} M \times P_{2}$ with $\partial\left(-\beta_{2} M \times D^{2}\right)$. There is a canonical map

$$
h: \bar{M} \longrightarrow \mathbf{C P}^{1}
$$

which sends $M \subset \bar{M}=M \cup\left(-\beta_{2} M \times D^{2}\right)$ to the point, and $\beta_{2} M \times D^{2}$ to $\mathbf{C P}^{1}=S^{2}$ by the composition

$$
\beta_{2} M \times D^{2} \longrightarrow D^{2} \longrightarrow D^{2} / S^{1}=\mathbf{C P}^{1} .
$$

The map $h$ composed with the inclusion $\mathbf{C P}^{1} \subset \mathbf{C P}^{\infty}$ gives the map $\bar{h}: \bar{M} \longrightarrow$ $\mathbf{C P}^{\infty}$, and, consequently, a linear complex bundle $\xi \longrightarrow \bar{M}$ which is trivialized over $M$. The Spin-structure on $M$ together with the linear bundle $\xi \longrightarrow M$ determines a $S p i n^{c}$-structure on $\bar{M}$. To choose a metric $g_{0}$ on the disk, We identify $D^{2}$ with the standard hemisphere $S_{+}^{2}$ with a small color attached to the circle $S^{1}$, so that the metric $\left.g_{0}\right|_{S^{1}}$ is the standard flat metric $d \theta^{2}$. Then we have the product metric $g_{\beta_{2} M} \times g_{0}$ on $\beta_{2} M \times D^{2}$. Together with the metric $g$ on $M$, it gives a psc-metric $\bar{g}$ on $\bar{M}$. We choose a $U(1)$-connection on the linear bundle $\xi \longrightarrow M$, and let $F$ be its curvature form. We notice that since $\xi$ is trivialized over $M \subset \bar{M}$, the form $F$ is supported only on the submanifold $-\beta_{2} M \times D^{2} \subset \bar{M}$. Moreover, we have defined the bundle $\xi \longrightarrow \bar{M}$ as a pullback from the tautological complex linear bundle over $\mathbf{C P}^{1}$. Thus locally we can choose a basis $e_{1}, e_{2}, \ldots e_{n}$ of the Clifford algebra, so that $F\left(e_{1}, e_{2}\right) \neq 0$, and $F\left(e_{i}, e_{j}\right)=0$ for all other indices $i, j$. Notice also that the scalar curvature function $R_{\bar{g}}=R_{g_{\beta_{2} M}}+R_{g_{0}}$. Let $D$ be the Dirac operator on the canonical bundle $S(\bar{M})$ of Clifford modules over $\bar{M}$. We have the BLW-formula

$$
D^{2}=\nabla^{*} \nabla+\frac{1}{4}\left(R_{\bar{g}}+R_{g_{0}}\right)+\frac{1}{2} F\left(e_{1}, e_{2}\right) \cdot e_{1} \cdot e_{2} .
$$


Now we scale the metric $g_{\beta_{2} M}$ to the metric $\epsilon^{2} \cdot g_{\beta_{2} M}$ with the scalar curvature $R_{\epsilon^{2} \cdot g_{\beta_{2} M}}=\epsilon^{-2} \cdot R_{g_{\beta_{2} M}}$. Clearly this scaling does not effect the connection form since the scaling is in the "perpendicular direction". Let $\epsilon>0$ be such that the term

$$
\frac{1}{4}\left(\epsilon^{-2} \cdot R_{g_{\beta_{2} M}}+R_{g_{0}}\right)
$$

will dominates the connection term $\frac{1}{2} F\left(e_{1}, e_{2}\right) \cdot e_{1} \cdot e_{2}$. Then we attach the cylinder $\beta_{2} M \times[0, a] \times D^{2}$ (for some $a>0$ ) with the metric $g_{\beta_{2} M}(t) \times g_{0}$, where

$$
g_{\beta_{2} M}(t)=\frac{a-t}{a} g_{\beta_{2} M}+\frac{t}{a} \epsilon_{\beta_{2} M}^{2}+d t^{2},
$$

so that the metric $g_{\beta_{2} M}(t) \times g_{0}$ has positive scalar curvature, and is a product metric near the boundary. Thus with that choice of metric, the right-hand side in (24) becomes positive, which implies that the Dirac operator $D$ is invertible, and hence $\operatorname{ind}(D) \in K_{*}$ vanishes. This completes the case of $\eta$-singularity.

Remark 8.3 Here the author would like to thank S Stolz for explaining this matter.

The case $\Sigma_{2}=\left(P_{1}, P_{2}\right)$ is just a combination of the above argument and the BLW-formula for $\operatorname{Spin}^{c} \mathbf{Z} / k$-manifolds given by Freed [5].

The last case, when $\Sigma=\Sigma_{3}=\left(P_{1}, P_{2}, P_{3}\right)$ there is nothing to prove since $K O^{\Sigma_{3}}$ is a contractible spectrum, and thus any $\Sigma_{3}$-manifold has a psc-metric. Indeed, we have that

$$
\Omega_{n}^{\text {Spin, }, \Sigma_{3}} \cong \operatorname{Im} T, \quad \text { if } \quad n \geq 17 .
$$

This completes the proof of Theorem 1.1.

\section{References}

[1] D W Anderson, E H Brown, F P Peterson, The structure of Spin cobordism ring, Ann. of Math. 86 (1967) 271-298

[2] N Baas, On bordism theory of manifolds with singularities, Math. Scand. 33 (1973) 279-302

[3] B Botvinnik, Manifold with singularities and the Adam-Novikov spectral sequence, Cambridge University Press (1992)

[4] B Botvinnik, P Gilkey, S Stolz, The Gromov-Lawson-Rosenberg conjecture for groups with periodic cohomology, J. Diff. Geom. 46 (1997) 374-405 
[5] D S Freed, Z/ $k$-Manifolds and families of Dirac operators, Invent. Math. 92 (1988) 243-254

[6] D S Freed, Two index theorems in odd dimensions, Comm. Anal. Geom. 6 (1998) 317-329

[7] D S Freed, R B Melrose, A mod $k$ index theorem, Invent. Math. 107 (1992) 283-299

[8] P Gajer, Riemannian metrics of positive scalar curvature on compact manifolds with boundary, Ann. Global Anal. Geom. 5 (1987) 179-191

[9] M Gromov,H B Lawson, The classification of simply connected manifolds of positive scalar curvature, Ann. Math. 11 (1980) 423-434

[10] M Gromov, H B Lawson, Positive scalar curvature and the Dirac operator on complete manifolds, Publ. Math. I.H.E.S. no. 58 (1983) 83-196

[11] N Higson, An approach to $\mathbf{Z} / k$-index theory, Int. J. of math. Vol. 1, No. 2 (1990) 189-210

[12] N Hitchin, Harmonic spinors, Advances in Math. 14 (1974) 1-55

[13] M J Hopkins, M A Hovey, Spin cobordism determines real $K$-theory, Math. Z. 210 (1992) 181-196

[14] J Kaminker, K P Wojciechowski, Index theory of $\mathbf{Z} / k$ manifolds and the Grassmannian, from: "Operator algebras and topology (Craiova, 1989)", Pitman Res. Notes Math. Ser. 270, Longman Sci. Tech. Harlow (1992) 82-92

[15] M Kreck, S Stolz, HP ${ }^{2}$-bundles and elliptic homology, Acta Math. 171 (1993) 231-261

[16] D Joyce, Compact 8-manifolds with holonomy Spin(7), Invent. Math. 123 (1996) 507-552

[17] M Mahowald, The image of $J$ in the EHP sequence, Ann. of Math. 116 (1982) 65-112

[18] M Mahowald, J Milgram, Operations which detect $S q^{4}$ in the connective K-theory and their applications, Quart. J. Math. 27 (1976) 415-432

[19] O K Mironov, Existence of multiplicative structure in the cobordism theory with singularities, Math. USSR Izv. 9 (1975) 1007-1034

[20] A Hassell, R Mazeo, R B Melrose, A signature formula for manifolds with corners of codimension two, Preprint, MIT (1996)

[21] J W Morgan, D Sullivan, The transversality characteristic classes and linking cycles in surgery theory, Ann. of Math. 99 (1974) 461-544

[22] J Rosenberg, Groupoid $C^{*}$-algebras and index theory on manifolds with singularities, arxiv:math.DG/0105085

[23] J Rosenberg, S Stolz, A "stable" version of the Gromov-Lawson conjecture, from: "The Cech centennial (Boston, MA, 1993)", Contemp. Math. 181, Amer. Math. Soc. Providence, RI (1995) 405-418, 
[24] J Rosenberg, S Stolz, Manifolds of positive scalar curvature, from: "Algebraic topology and its applications", Math. Sci. Res. Inst. Publ. 27, Springer, New York (1994) 241-267,

[25] J Rosenberg, S Stolz, Metrics of positive scalar curvature and connections with surgery, to appear in "Surveys on Surgery Theory", vol. 2, Ann. of Math. Studies, vol. 149

[26] T Schick, A counterexample to the (unstable) Gromov-Lawson-Rosenberg conjecture, Topology 37 (1998) 1165-1168

[27] R Schoen, S T Yau, On the structure of manifolds with positive scalar curvature, Manuscripta Math. 28 (1979) 159-183

[28] D Sullivan, Triangulating and smoothing homotopy equivalence and homeomorphisms, Geometric topology seminar notes, Princeton University Press (1967)

[29] S Stolz, Simply connected manifolds of positive scalar curvature, Ann. of Math. 136 (1992) 511-540

[30] S Stolz, Splitting of certain MSpin-module spectra, Topology, 133 (1994) 159180

[31] S Stolz, Concordance classes of positive scalar curvature metrics, to appear

[32] R E Stong, Notes on Cobordism Theory, Princeton University Press (1968)

[33] U Würgler, On the products in a family of cohomology theories, associated to the invariant prime ideal of $\pi_{*}(B P)$, Comment. Math. Helv. 52 (1977) 457-481

[34] W Zhang, A proof of the mod 2 index theorem of Atiyah and Singer, C. R. Acad. Sci. Paris Sér. I Math. 316 (1993) 277-280

[35] W Zhang, On the mod $k$ index theorem of Freed and Melrose, J. Differential Geom. 43 (1996) 198-206 